\title{
Stationary Equilibrium Transition Rules for an Overlapping Generations Model with Uncertainty*
}

\author{
JOHN P. LAITNER \\ Department of Economics, University of Michigan, \\ Ann Arbor, Michigan 48109
}

Received October 5, 1983; revised July 15, 1984

\begin{abstract}
An overlapping generations model incorporating random production shocks is studied. Households have finite life spans. Futures markets are incomplete. Agents have full information in one case, and receive only a limited signal in another. In both instances the existence of a time-autonomous transition rule is proved such that if all agents forecast using it, the economy's actual growth will bear the predictions out. The rule corresponds to a steady state for a non-stochastic model. With limited information, it is seen to depend on all past signals. Several approximation theorems which may facilitate future applications are presented. Journal of Economic Literature Classification Numbers: $021,026,111,131$. 1985 Academic Press, Inc.
\end{abstract}

\section{INTRODUCTION}

There are a variety of possible approaches for studying the equilibria over time of economies which are subject to random disturbances. One method presupposes that a permanent central authority (or single infinitely lived household) manages key variables to maximize an economywide welfare function-see Brock and Mirman [4] and Merton [14]. (Recent applications and elaborations include, for example, Danthine and Donaldson [5] and Kydland and Prescott [12].) A potential second alternative is the Arrow-Debreu model $[1,6]$, which is not centrally directed. Bewley's [3] extension of it to an infinite number of goods allows the possibility of studying economic growth over a limitless time horizon by labelling commodities according to their periods.

A third approach also assumes a decentralized cconomy, but in contrast to Arrow and Debreu restricts contracting to a limited set of markets operating at discrete intervals. Analyses in this class are sometimes called "temporary equilibrium models"-see, for instance, $[11,1,10,17]$. Often

* I owe thanks to my colleagues T. Bergstrom and C. Simon for helpful comments on an earlier draft of this work. This research was supported by the National Science Foundation. Grant SES-8106555. 
agents in such models can trade current goods freely but have only a few financial instruments available for their savings-perhaps because in practice the costs of setting up and operating markets serve to reduce their numbers. ${ }^{1}$ Because of the restricted and sequential nature of opportunities for transactions, agents generally need to predict the future behavior of others.

This paper studies a model from the third group. We use a variant of the life-cycle savings paradigm familiar from $[18,7,19]$, modified to incorporate stochastic shocks. The economy is decentralized and it lasts foreveralthough individual households do not. We assume that all agents at each time possess the same information and that their expectations about the future are rational in the sense defined by Muth [16]. We consider two versions of the framework, one with complete information, and one in which agents are incompletely informed.

Our principal contribution is to establish the existence of time-autonomous probability transition rules determining growth paths which are sequences of temporary equilibria consistent with rational expectations. Because of their time independence, the transition functions are natural stochastic analogues of deterministic steady states. They belong to the category of solutions which Hicks [11] calls "equilibria over time" and which Radner [17] labels "equilibria of plans, prices, and price expectations" (as well as "the perfect foresight approach"). The connection between utility and production functions, on the one hand, and our equilibrium transition rules, on the other, is explicit.

In the full information version of the model, our equilibrium generates a Markov process for vectors of state variables. The process has a finite number of stationary probability distributions, and the economy must begin to obey one within a finite number of time periods. We present a mathematical result on the feasibility of obtaining practical approximations of our equilibrium transition rule.

The analysis with limited information has somewhat different characteristics. Perhaps because of the costs and other difficulties of acquiring personal asset and income data on households, in this case at each date our agents receive signals which only partially reveal the current state of the economy. In such a world, we show that market participants will generally find old signals useful for reducing their uncertainty about the present. In fact, our equilibrium transition rule depends on information from the infinite past. A crucial question is whether signals from the very distant past have an asymptotically small importance, and we outline a test for that property. Again, we also discuss approximations.

\footnotetext{
${ }^{1}$ Also, at any given time, not all of the parties with whom a current agent will expect someday to conduct trades will yet have been born.
} 
We make very few assumptions about preferences and production. In order to establish the existence of our two equilibrium transition rules, however, we do require that all commodity magnitudes and price levels come from discrete sets. Although this at first may seem mathematically less elegant than employing continuous variables, it enables us to obtain results which otherwise apparently (see Sect. 2) would be out of reach or would require very detailed economic behavioral restrictions. It also provides a framework which appears to be very flexible with regard to admitting elaborations beyond the scope of this paper. Arguably costs of measurement precision, record storage, and computation do lead to discreteness in all actual economies.

The organization of this paper is as follows. Section 1 presents our basic model. Section 2 proves the existence of a stationary equilibrium transition rule in the case of full information. Section 3 considers approximations. Section 4 studies the case of limited information.

\section{Discrete Variables}

This section presents our full information model.

Our household sector consists of overlapping generations of families. Each family lives $N$ periods. We let $2 \leqslant N<\infty$. If $N \geqslant 3$, at each time there will be age-related differences among households young enough to save; consequently, with virtually no sacrifice of generality, we can disregard other sources of intragenerational heterogeneity and let there be one family of each age alive in each period. There are no inheritances or bequests. There is no government sector. Physical capital is the sole outlet for saving. As in Brock and Mirman [4], there is an aggregate production function subject to random shocks. Although the overall framework is reminiscent of a stochastic version of Diamond [7], Section 2 shows that the fact that we allow family life spans longer than two periods is extremely important (in our context).

As stated in the introduction, we assume all price and quantity variables belong to discrete sets. Section 2 attempts to illuminate the need for this. We assume each discrete set contains all non-negative integral multiples of a minimal unit. Prices, for example, may all be whole numbers of pennies. Let $I$ be the non-negative integers.

The detailed elements of our model are as follows.

\section{Production}

If $Q_{t}$ is aggregative output at time $t, K_{t}$ the aggregative (physical) capital stock, $L$ the potential labor supply, and $\lambda_{t}$ a realization from the random 
shock variable $\tilde{\lambda}$, the economy has an aggregate production function $f(\cdot)$ with

$$
Q_{t}=f\left(K_{t}, L, \lambda_{t}\right)
$$

Capital depreciates in one period, and $K_{t}$ must have been built (and financed) at time $t-1$. We normalize the price of output to 1 and assume it is homogeneously divisible into investment and consumption goods. If $l_{j} \in I$ is the (exogenously given) labor supply of the household of age $j$ (for any time $t$ ),

$$
L=\sum_{j=1}^{N} l_{j}
$$

For the sake of simplicity, we let $\lambda_{t}, \lambda_{t+1}, \ldots$ be independent samplings from $\tilde{\lambda}$-which has a known distribution. We want $f(\cdot)$ to obey an Inada condition creating an upper bound (see Lemma 1 ) for the sustainable magnitude of $K_{t}$, and we let $f(\cdot)$ be monotone in $K$ and $L$. Formally,

\section{(A1) We assume}

(i) For all $t, \lambda_{t} \in A$ with $A$ a finite set. $p(\lambda)$ gives the probability of each realization of $\tilde{\lambda}$.

(ii) $f: I \times I \times A \rightarrow I$ is nondecreasing in its first two arguments.

(iii) There exists $\hat{K} \in I$ with $\hat{K} \geqslant 1$ such that $\lambda \in A, K \in I$, and $K \geqslant \hat{K}$ imply $f(K, L, \lambda)<K$.

\section{The Labor Market}

Aside from price and quantity discreteness, we want the labor market to operate along neoclassical lines. Let $W_{t}$ be the time $t$ wage rate.

The correspondence giving the aggregate demand for labor at time $t$ is

$$
\begin{aligned}
\mathscr{L}\left(K_{t}, W_{t}, \lambda_{t}\right) \equiv\left\{L^{*}\right. & \in I \cup\{\infty\} \mid f\left(K_{t}, L^{*}, \lambda_{t}\right)-W_{t} \cdot L^{*} \\
& \left.=\sup _{L^{* *} \in I \cup|\infty|}\left\{f\left(K_{t}, L^{* *}, \lambda_{t}\right)-W_{t} \cdot L^{* *}\right\}\right\} .
\end{aligned}
$$

Because $W_{t}$ must be an integer, we can not expect in general that labor supply and demand will equal one another exactly. Instead, we assume $W_{t}$ always takes the highest value consistent with zero or positive excess labor demand. Imagine, for example, that competition for employment immediately eliminates any wage leaving an excess labor supply. ${ }^{2}$

\footnotetext{
${ }^{2}$ As our proofs show, many other assumptions, here and in the case of profit distributions, are possible.
} 
We want a lower bound, $\check{K}$, for the capital stock such that a market wage exists for all $K \geqslant \check{K}$ and, to make a positive labor supply credible, such that $\sup \mathscr{L}(K, 1, \lambda) \geqslant L$ all $K \geqslant \check{K}$ and $\lambda \in A$. Section 2 establishes the existence of equilibria with $K_{t} \geqslant \breve{K}$ all $t$.

Define

$$
\mathscr{W}\left(K_{t}, \lambda_{t}\right) \equiv\left\{W_{t} \in I \mid \sup \mathscr{L}\left(K_{t}, W_{t}, \lambda_{t}\right) \geqslant L\right\}
$$

Then

(A2) We assume there exists $\check{K} \in I$ such that $\check{K}<\hat{K}$ and $1 \in \mathscr{W}(K, \lambda)$ all $\lambda \in A$ and $K \in I$ with $K \geqslant \check{K}$. If $\mathscr{W}\left(K_{t}, \lambda_{t}\right) \neq \varnothing$, total employment is $L$ and the market wage is $W_{t}=W\left(K_{t}, \lambda_{t}\right) \equiv \sup \mathscr{W}\left(K_{t}, \lambda_{t}\right)$.

\section{Assets and Profits}

As in the case of labor, we want returns for savers to correspond closely to neoclassical pricing.

Let $a_{j t}$ be the assets the family of age $j$ at time $t$ carries from $t$ to $t+1$, let $a_{t} \equiv\left(a_{1 t}, \ldots, a_{N t}\right)$, and let $l$ be a row vector of ones. Then $K_{t+1}=t a_{t}$. The absence of bequests implies $a_{N t}=0$ at $t$. We assume bankruptcy laws prevent families from ever having negative net worth:

(A3) We assume $a_{t} \in I^{N}$ all $t$.

We must face the following minor complication. If total profits are $\pi_{t}$,

$$
\pi_{t}=\pi\left(K_{t}, \lambda_{t}\right)=f\left(K_{t}, L, \lambda_{t}\right)-W\left(K_{t}, \lambda_{t}\right) \cdot L .
$$

Suppose $\pi_{t}=\$ 1.01$ and $K_{t}=3$. Then an equal, integral distribution of profits is impossible. The next assumption requires that in such a situation two units of capital receive $\$ .34$ and one receives $\$ .33$, and that all three outcomes satisfying these conditions are equally likely.

If we definc

$$
r\left(K_{t}, \lambda_{t}\right) \equiv \max \left\{r^{*} \in I \mid r^{*} K_{t} \leqslant \pi\left(K_{t}, \lambda_{t}\right)\right\},
$$

$r\left(K_{t}, \lambda_{t}\right)$ corresponds to $\$ .33$ in our arithmetic example. Then

(A4) Given $K_{t} \geqslant \check{K}$ and $\lambda_{t} \in A$, we assume that all profit distributions exhausting $\pi\left(K_{t}, \lambda_{t}\right)$ and paying each asset unit either $r\left(K_{t}, \lambda_{t}\right)$ or $1+$ $r\left(K_{t}, \lambda_{t}\right)$ are possible and equally likely. No other distributions occur.

Suppose $a_{t-1} \in I^{N}, K_{t}=a_{t-1} \geqslant \check{K}$, and $\lambda_{t} \in \Lambda$. Name the (finite) set of acceptable time $t$ profit distributions $\Omega^{*}\left(K_{t}, \lambda_{t}\right)$. Let the (equal) ex ante chance of each one be $p^{*}\left(K_{t}, \lambda_{t}\right)$. Then if $y_{j t}$ is the total time $t$ income of family $j$-including $l_{j}, W\left(K_{t}, \lambda_{t}\right)$ as well as profits-and if $y_{t} \equiv\left(y_{1 t}, \ldots, y_{N t}\right)$, 
we can define a function $\Phi(\cdot)$ such that conditional on any $\omega_{t}^{*} \in$ $\Omega *\left(l a_{t-1}, \lambda_{t}\right)$

$$
y_{t}=\Phi\left(a_{t-1}, \lambda_{t}, \omega_{t}^{*}\right)
$$

\section{Household Preferences}

In order to employ dynamic programming below, we assume that household utility functions are additively separable.

(A5) We assume

(i) if a given household's age $i$ consumption is $c_{i} \geqslant 0$, its current flow of utility is a finite real number $u\left(c_{i}, i\right)$.

(ii) At age $j$ the household above chooses its saving and consumption to maximize, subject to our non-negativity constraint for asset holdings, the expected value of $\sum_{i=j}^{N} u\left(c_{i}, i\right)$.

\section{Household Forecasts}

We assume (until Section 4) that households know at each time $t$ the current distribution of income, $y_{t}$. This vector should register all data that agents need for predicting $a_{t} ; a_{t}$, in turn, implies (via $t a_{t}=K_{t+1}$ ) an ex ante distribution for next-period factor prices and for $y_{t+1}$, on which forecasts farther into the future depend. Let $\mathscr{P}\left(I^{N}\right)$ be the power set of $I^{N}$. Then for every $A_{t} \in \mathscr{P}\left(I^{N}\right)$, we use a probability transition rule

$$
g\left(A_{t}, y_{t}\right) \equiv \operatorname{Pr}\left\{a_{t} \in A_{t} \mid y_{t}\right\}
$$

to describe each family's anticipations about $a_{t}$ conditional on $y_{t}$. Because of our discrete variables, even if utility functions are strictly concave, households will sometimes find themselves indifferent between several savings levels. Other than that, $a_{t}$ would always follow deterministically from $y_{t}$; because of it, sensible expectation functions $g(\cdot)$ may well assign positive probabilities to more than one $a_{t}$ for some vectors $y_{t}$.

Notice that assumption (A1) implies the economy cannot maintain a capital stock exceeding $\hat{K}$ :

LemmA 1. Suppose assumption (A 1) holds. Let $a_{t} \in I^{N}$ be the economy's actual vector of assets of the close of period $t$. Then if $t a_{t}>\hat{K}$, there exists $t^{\prime}>t$ such that $i a_{t^{\prime \prime}} \leqslant \hat{K}$, all $t^{\prime \prime} \geqslant t^{\prime}$. If $i a_{t} \leqslant \hat{K}, i a_{t^{*}} \leqslant \hat{K}$ all $t^{*} \geqslant t$.

The Appendix presents a proof.

In light of Lemma 1, we confine our attention to states of the economy with $l a_{t-1} \leqslant \hat{K}$. In fact, to insure that $W_{t}$ is well defined, we concentrate (with the help of assumption (A7)) on values of $a_{t-1}$ in the finite set

$$
\mathscr{A} \equiv\left\{a \in I^{N} \mid \check{K} \leqslant l a \leqslant \hat{K}\right\},
$$


on values of $y_{t}$ in (the finite set)

$$
\begin{aligned}
& \mathscr{Y} \equiv\left\{y \in I^{N} \mid \text { for some } a_{t-1} \in \mathscr{A}, \lambda_{t} \in \Lambda,\right. \text { and } \\
& \left.\qquad \omega_{t}^{*} \in \Omega^{*}\left(l a_{t-1}, \lambda_{t}\right), y=y_{t}=\Phi\left(a_{t-1}, \lambda_{t}, \omega_{t}^{*}\right)\right\},
\end{aligned}
$$

and on expectation rules in

$$
\begin{array}{r}
\xi \equiv\{g: \mathscr{P}(\mathscr{A}) \times \mathscr{Y} \rightarrow[0,1] \mid \text { for each } y \in \mathscr{Y}, \\
g(\cdot, y) \text { is a probability measure on } \mathscr{A}\} .
\end{array}
$$

Note that the conditions $g \in \mathscr{F}$ must obey for all $(A, y) \in \mathscr{P}(\mathscr{A}) \times \mathscr{Z}$ are $0 \leqslant g(A, y) \leqslant 1, g(\mathscr{A}, y)=1$, and $g(A, y)=\sum_{a \in A} g(\{a\}, y)$. We often write $g(a, y)$ in place of $g(\{a\}, y)$.

Suppose household $j$ observes $y_{t} \in \mathscr{Z}$ and forecasts future factor returns using $g\left(\cdot, y_{t}\right)$ some $g \in \mathcal{F}$. Let $x_{j t}$ be its actual saving at time $t$. Our definition of "equilibrium" below forces $x_{j t}$ and predictions of $a_{j t}$ via $g\left(\cdot, y_{t}\right)$ to correspond in a precise manner. For the time being, however, we allow the two to be completely distinct so that, as in conventional competitive models, household $j$ can believe that its choice of $x_{j t}$ has no noticeable effect on overall prices.

Consider the returns household $j$ projects (at time $t$ ) for units of $x_{j t}$. Conditional on any $a_{t} \in \mathscr{A}, \quad K_{t+1}=\imath a_{t}, \quad \lambda_{t+1} \in A, \quad$ and $\omega_{t+1}^{*} \in$ $\Omega^{*}\left(K_{t+1}, \lambda_{t+1}\right)$, if $x_{j t}=a_{j t}$, the household should anticipate a time $(t+1)$ income equal to component $j+1$ of $\Phi\left(a_{t}, \lambda_{t+1}, \omega_{t+1}^{*}\right)$. If $x_{j t} \neq a_{j t}$, we assume the household will anticipate a return on each extra unit of savings of $1+r\left(K_{t+1}, \lambda_{t+1}\right)$ with an ex ante probability $\rho\left(K_{t+1}, \lambda_{t+1}\right)$-equaling the frequency of this return in the economy (given $K_{t+1}$ and $\lambda_{t+1}$ ),

$$
\rho\left(K_{t+1}, \lambda_{t+1}\right) \equiv\left[\pi\left(K_{t+1}, \lambda_{t+1}\right)-r\left(K_{t+1}, \lambda_{t+1}\right) \cdot K_{t+1}\right] / K_{t+1}
$$

and a return of $r\left(K_{t+1}, \lambda_{t+1}\right)$ with probability $1-\rho\left(K_{t+1}, \lambda_{t+1}\right)$. Again this seems consistent with the usual idea of price-taking behavior.

Formally,

(A6) we assume

(i) all households forecast with the same expectation function $g \in \mathscr{F}$.

(ii) At time $t$, conditional on $a_{t} \in \mathscr{A}, K_{t+1}=\imath a_{t}, \lambda_{t+1} \in A$, and $\omega_{t+1}^{*} \in \Omega^{*}\left(K_{t+1}, \lambda_{t+1}\right)$, household $j$ will anticipate a time $(t+1)$ income equal to component $j+1$ of $\Phi\left(a_{t}, \lambda_{t+1}, \omega_{t+1}^{*}\right)$ if its current savings, $x_{j t}$, equal $a_{j t}$. Let $r=r\left(K_{t+1}, \lambda_{t+1}\right)$ and $\rho=\rho\left(K_{t+1}, \lambda_{t+1}\right)$. It will anticipate a gain (loss) on each unit by which $x_{j t}>(<) a_{j t}$ of $1+r$ with probability $\rho$, and $r$ with probability $1-\rho$-the outcomes for different units of $x_{j t}-a_{j t}$ being independent. 
Using the notation of (A6), let the set of anticipated possible payoffs on $x_{j t}-a_{j t}$ at $t+1$ be

$$
\Omega^{* *}=\Omega^{* *}\left(a_{t}, \lambda_{t+1}, x_{j t}, j\right),
$$

label the probability (computed from (A6)) of each $\omega_{t+1}^{* *} \in \Omega^{* *}$ with $p^{* *}\left(a_{t}, \lambda_{t \mid 1}, x_{j t}, \omega_{t+1}^{* *}, j\right)$, and let the family's total next-period income given $\omega_{t+1}^{* *} \in \Omega^{* *}$ be $\phi\left(a_{t}, \lambda_{t+1}, x_{j t}, \omega_{i+1}^{*}, \omega_{t+1}^{* *}, j\right)$.

\section{Household Behavior}

We now turn to the utility-maximization problem of households.

If family $j$ at time $t$ uses $g \in \mathscr{G}$ and $y_{t} \in \mathscr{Y}$ to forecast the future and has an actual present income $y_{j t}^{*}$, let $v\left(y_{j t}^{*}, y_{t}, j ; g\right)$ be its expected lifetime utility from time $t$ onward. We define $v: I \times \mathscr{Y} \times\{1, \ldots, N\} \times \mathscr{G} \rightarrow(-\infty, \infty)$ recursively: for $j=N$,

$$
v\left(y_{N t}^{*}, y_{t}, N ; g\right)=u\left(y_{N t}^{*}, N\right)
$$

for $1 \leqslant j \leqslant N-1$,

$v\left(y_{j t}^{*}, y_{t}, j ; g\right)$

$$
\begin{aligned}
= & \max _{\left\{x \in I: x \leqslant y_{j t}^{*}\right\}}\left\{u\left(y_{j t}^{*}-x, j\right)+\sum_{a_{t} \in \mathscr{A}, \lambda_{t+1} \in \Lambda} g\left(\left\{a_{t}\right\}, y_{t}\right) \cdot p\left(\lambda_{t+1}\right)\right. \\
& \cdot \sum_{\omega_{t}^{*} \in \Omega^{*}\left(t a_{t}, \lambda_{t+1}\right\}, \omega_{t+1}^{* *} \in \Omega^{* *}\left(a_{t}, \lambda_{t+1}, x, j\right)} p^{*}\left(t a_{t}, \lambda_{t+1}\right) \\
& \cdot p^{* *}\left(a_{t}, \lambda_{t+1}, x, \omega_{t+1}^{* *}, j\right) \\
& \left.\cdot v\left(\phi\left(a_{t}, \lambda_{t+1}, x, \omega_{t+1}^{*}, \omega_{t+1}^{* *}, j\right), \Phi\left(a_{t}, \lambda_{t+1}, \omega_{t+1}^{*}\right), j+1 ; g\right)\right\} .
\end{aligned}
$$

Lines (14), (15) determine each family's saving at each age $j$.

Let $\psi\left(y_{j t}^{*}, y_{t}, j ; g\right)$ be the set of maximizing values of $x$ in line (15). Because of our discrete units, such a set may contain more than one element. Since there are no bequests, $\psi\left(y_{N t}^{*}, y_{t}, N ; g\right)=\{0\}$. Define

$$
\Psi\left(y_{t} ; g\right) \equiv \psi\left(y_{1 t}, y_{t}, 1 ; g\right) \times \cdots \times \psi\left(y_{N t}, y_{t}, N ; g\right) .
$$

Then for each $g \in \mathscr{F}$ and $y_{t} \in \mathscr{Y}, \Psi\left(y_{t} ; g\right)$ gives all desired savings vectors $\left(x_{1 t}, \ldots, x_{N t}\right) \equiv x_{t}$. In each case, we can see that $\Psi\left(y_{t} ; g\right) \neq \varnothing$ and $\Psi\left(y_{t} ; g\right) \subset I^{N}$.

If $y_{t} \in \mathscr{Y}$, we have

$$
\imath a_{t} \leqslant y_{t} \leqslant \max _{\lambda \in \Lambda} f(\hat{K}, L, \lambda)<\hat{K} .
$$


Thus, for $y_{t} \in \mathscr{Z}$ and $g \in \mathscr{F}$,

$$
x_{t} \in \Psi\left(y_{t} ; g\right) \quad \text { implies } \quad l x_{t}<\hat{K}
$$

The next assumption guarantees that $l x_{t} \geqslant \check{K}$ as well.

(A7) If $y_{t} \in \mathscr{Y}$ and $g \in \mathscr{F}$, we assume $l x_{t} \geqslant \check{K}$ all $x_{t} \in \Psi\left(y_{t} ; g\right)$.

The idea is as follows. Suppose $g\left(\cdot, y_{t}\right)$ assigns substantial probability to next-period capital stocks implying high interest and low wage rates. Then all age groups will tend to want to save to take advantage of favorable returns and to protect themselves against low next-period wages. If $g\left(\cdot, y_{t}\right)$ heavily weights the opposite outcome, people on the verge of retirement will want to save a great deal to defend themselves in the event of low interest rates. At the cost of more notation, we could allow personal uncertainties about incapacitating illness and skill obsolescence. As in the case of imminent retirement, households vulnerable to these problems would tend to save heavily (whether directly or in the form of insurance premiums) in the face of projected low returns. Parenthetically, extreme capital depletion does not seem to be a significant issue in, for example, Tobin's [19] detailed and empirically motivated model. Assumption (A7) rules it out here.

For $g \in G$ define

$$
\begin{aligned}
& \Gamma(g) \equiv\left\{\gamma: \mathscr{P}(\mathscr{A}) \times \mathscr{Y} \rightarrow[0,1] \mid \text { for all } y_{t} \in \mathscr{Y},\right. \\
& \\
& \gamma\left(\cdot, y_{t}\right) \text { is a probability measure on } \mathscr{A} \text { with } \\
&\left.\gamma\left(\left\{a_{t}\right\}, y_{t}\right)=0 \text { each } a_{t} \notin \Psi\left(y_{t} ; g\right)\right\} .
\end{aligned}
$$

Then $\Gamma(g)$ gives the set of possible actual transition rules for the economy if all agents maximize utility and use the expectation rule $g \in \mathscr{G}$. Because assumptions (A1) and (A7) guarantee

$$
\Psi(y ; g) \subset \mathscr{\&} \quad \text { all } y \in \mathscr{Y}, g \in \mathscr{G},
$$

$\Gamma(g)$ includes all such rules and we have

$$
\Gamma(g) \neq \varnothing \quad \text { each } \quad g \in \mathscr{G} .
$$

Comparing lines (11) and (19),

$$
\Gamma(g) \subset \mathscr{G} \text { each } g \in \mathscr{G} .
$$

$\Gamma(g)$ will be a set with more than one element if $\Psi(y ; g)$ is for any $y \in \mathscr{Y}$. 


\section{EQUILIBRIUM WITH FULL INFORMATION}

This section defines our concept of equilibrium with full information and establishes existence. We also show that each equilibrium induces a Markov process for $a_{t}$ on $\mathscr{A}$ and that the process has a finite number of ergodic sets and stationary distributions, and we try to explain the importance of discrete variables in our analysis.

Our definition of equilibrium is

Definition. $g(\cdot)$ is a "stationary equilibrium transition function" if $g \in$ $\mathscr{E} \equiv\{g \in \mathscr{G} \mid g \in \Gamma(g)\}$.

The word "stationary" refers to the fact that each $g \in \mathscr{F}$ is time autonomous. The word "equilibrium" refers to the facts (1) that throughout our analysis we insist that markets clear (in accordance with (A2) and (A4)) and (2) that expectations are rational for each $g \in \mathscr{E}$ in the sense that if all agents forecast with $g$, actual growth-specified by $\Gamma(g)$-can bear out their anticipations (about possible future prices and corresponding probabilities). If at least one consumer is equally satisfied satisfied with several current consumption-saving strategies, $g \in \Gamma(g)$ implies $g(\cdot)$ is consistent with one of them or with some ex ante assignment of probabilities to a subset of them.

Despite the discrete-variable domain of each $g(\cdot)$, fortunately the set of functions $\mathscr{G}$ is convex. For $g, g^{\prime} \in \mathscr{G}$, define

$$
\left\|g-g^{\prime}\right\| \equiv \max \left\{\left|g(\{a\}, y)-g^{\prime}(\{a\}, y)\right|:(a, y) \in \mathscr{A} \times \mathscr{Z}\right\} .
$$

Then

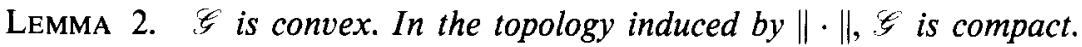
The Appendix provides a proof.

Lemma 2 enables us to establish the existence of a stationary equilibrium transition function.

Proposition 1. If assumptions (A1)-(A7) hold, the set $\mathscr{E}$ is non-empty. The Appendix supplies a proof. The proof applies a fixed point theorem to $\Gamma(\cdot)$ defined on $\mathscr{G}$.

Suppose $g \in \mathscr{E}$ determines the economy's evolution over time. Then for each $a_{t} \in \mathscr{A}$, the probability of transiting to $A_{t+1} \in \mathscr{P}(\mathscr{A})$ is $T\left(A_{t+1}, a_{t} ; g\right)$ with

$$
\begin{aligned}
T\left(A_{t+1}, a_{t} ; g\right)= & \sum_{\lambda_{t+1} \in \Lambda} \sum_{\omega_{t+1}^{*} \in \Omega^{*}\left(t a_{t} \cdot \lambda_{t+1}\right)} g\left(A_{t+1}, \Phi\left(a_{t}, \lambda_{t+1}, \omega_{t+1}^{*}\right)\right) \\
& \cdot p^{*}\left(t a_{t}, \lambda_{t+1}\right) \cdot p\left(\lambda_{t+1}\right) .
\end{aligned}
$$


For $g \in \mathscr{E}$, line (17) and assumption (A7) imply

$$
T(\mathscr{A}, a ; g)=1 \quad \text { all } a \in \mathscr{A} .
$$

Thus, $T(\cdot)$ defines a Markov process on $\mathscr{A}$.

An ergodic set for $T(\cdot ; g)$ will be a minimal subset $A \subset \mathscr{d}$ with

$$
T(A, a ; g)=1 \quad \text { all } a \in A .
$$

No two ergodic sets can overlap. Once the economy enters an ergodic set, it remains in it with probability 1 for each subsequent period. We have

Proposition 2. Let assumptions (A1)-(A7) hold and let $g \in \mathscr{E}$. Then $T(\cdot ; g)$ has a positive, finite number of ergodic sets in $\mathscr{H}$. Each ergodic set has a unique stationary measure, giving the long-term frequency distribution for all subsets of points within the set. With probability 1 the economy will enter some ergodic set within a finite number of time periods.

Once we note the finite number of points in $\mathscr{A}$, Proposition 2 follows directly from Theorems 2.3 and 2.4 in Chapter $V$ of Doob [8].

In closing this section, we can ask how essential discrete units are to our results-discreteness does after all complicate the presentation in Section 1. As an answer, consider the continuous-variable model in Laitner [13]. It is analogous to ours, except that families are restricted to two-period lives. With continuous variables, to obtain compactness for (with a metric corresponding to line (23)) we need equicontinuity: at each $(\{a\}, y)$ with $(a, y) \in \mathscr{A} \times \mathscr{Y}$, every function in $\mathscr{Z}$ must obey a maximal "steepness" condition (for changes in $y$ ). Once we limit $\mathscr{G}$ to an equicontinuous family, it is difficult to show $\Gamma(\mathscr{G}) \subset \mathscr{G}$. Laitner [13] proceeds with reliance on twoperiod life spans and special assumptions not needed in the present paper. With two-period lives, families only need to predict next period's aggregate capital stock; with $N>2$, they need to predict the whole next-period distribution of asset holdings - in order to be able to go on and forecast later factor prices. For $\Gamma(\mathscr{G}) \subset \mathbb{G}$ with two-period lives, we must show that if a change in $y_{t}$ leads to a moderate change in the predicted $K_{t+1}$, the actual value of $t a_{t}$ will vary only moderately; with $N>2$, a moderate change in the predicted $a_{t}$ may lead to a less moderate variation in the $K_{t+2}=\imath a_{t+1}$ predicted from $a_{t}$ (via $y_{t+1}$ ), causing the actual $a_{t}$ to change appreciably. For these reasons, in the absence of very special restrictions, Proposition 1 seems out of reach with $N>2$ and continuous variables. Constraining family lives to two periods, on the other hand, seems unsatisfactory. A further benefit of our approach is that the mathematics underlying this paper appear to allow many generalizations in addition to $N>2$. 


\section{Approximations}

Section 2 proves the existence of a fixed point for a correspondence defined over a space of functions. We now briefly indicate how an approximate equilibrium might be constructed.

Our economic model determines and $\Gamma(\cdot)$. We want $g \in \mathscr{F}$ with $g \in \Gamma(g)$. Define

$$
\Delta(g) \equiv \inf \left\{\left\|g-g^{\prime}\right\|: g^{\prime} \in \Gamma(g)\right\}
$$

all $g \in \mathscr{G}$. One natural approach is to try to minimize $\Delta(\cdot)$ over a restricted class in $\mathscr{G}$. We have

Proposition 3. Suppose $g_{m} \in \mathscr{G}$ all $m \in I$ and $\Delta\left(g_{m}\right) \rightarrow 0$ as $m \rightarrow \infty$. Then there exists $g \in \mathscr{E}$ and a subsequence $g_{n}$ of $g_{m}$ such that $\left\|g_{n}-g\right\| \rightarrow 0$ as $n \rightarrow \infty$.

The Appendix supplies a proof.

Suppose $\mathscr{G}^{m}$ is a subset of $m$-parameter functions in $\mathscr{F}$. Let $g_{m}$ minimize $\Delta(\cdot)$ on $\mathscr{G}^{m}$. (Notice that computing $g_{m}$ is a standard minimization problem on $R^{m}$-although $\Delta(\cdot)$ may well not be continuous.) Then if $\lim _{m \rightarrow \infty} \Delta\left(g_{m}\right)=0$, the sequence $g_{m}$ has a limit point $g \in \mathscr{E}$.

\section{LiMITED INFORMATION}

As outlined in the introduction, the ideas of incomplete financial markets and of limited access to information for all agents have related appeal from a practical standpoint. We now add the latter assumption to our framework.

Specifically, we let agents receive a signal $s_{t}$ at each time $t$ revealing current national accounts data on $K_{t}, W_{t}, L$, and $Q_{t}$. All previous signals also are public information. On the other hand, unlike Sections 1 and 2, agents do not observe distributions of assets or incomes in the economy at any date. ${ }^{3}$ We again prove the existence of a rational expectations equilibrium. Its form, however, is quite different from Section 2.

\section{The Framework}

We need to change the arguments of our household expectation function $g(\cdot)$ and to incorporate a related function $h(\cdot)$. Although we also need some new notation, many of the economic ideas of Sections 1 and 2 carry over. Assumptions (A1)-(A6) remain in force.

\footnotetext{
${ }^{3}$ Agents know their own income and asset holdings. However, as in conventional competitive models, we assume that this information alone about distributions has no bearing on economywide signals.
} 
The latter assumptions define a function $F(\cdot)$ giving $s_{t}$ for each $\left(a_{t-1}, \lambda_{t}\right) \in$ of $\times \Lambda$ :

$$
s_{t}=F\left(a_{t-1}, \lambda_{t}\right)
$$

Agents know the form of $F(\cdot)$. Let $f$ be the space of signals,

$$
\therefore=F(\mathscr{\mathscr { L }}, A) \text {. }
$$

Then $f$ is a finite set.

Consider the family of age $j$ at time $t$. Conditional on $s_{t+1}$ and its current savings choice $a_{j t}$, it can use (A2)-(A6) to analyze its time $(t+1)$ income possibilities. Index the household's next-period income possibilities set with $\alpha\left(a_{j t}, s_{t+1}\right)$, label the ex ante probability of each $\alpha_{t+1} \in \alpha\left(a_{j t}, s_{t+1}\right)$ with $q\left(a_{j t}, s_{t+1}, \alpha_{t+1}\right)$, and let the income figure corresponding to $\alpha_{t+1}$ be

$$
y_{j+1, t+1}=\mu\left(a_{j t}, s_{t+1}, \alpha_{t+1}\right) \in I .
$$

Conditional on knowledge of $s_{t+1}$ and the whole vector $a_{t}$, provided $l a_{t}$ and the $K_{t+1}$ component of $s_{t+1}$ coincide, the same household could deduce a set $\beta\left(a_{t}, s_{t+1}\right)$, a function $\mu^{*}(\cdot)$ with

$$
y_{t+1}=\mu^{*}\left(a_{t}, s_{t+1}, \beta_{t+1}\right) \in I^{N} \quad \text { each } \beta_{t+1} \in \beta\left(a_{t}, s_{t+1}\right),
$$

and a probability $q^{*}\left(a_{t}, s_{t, 1}, \beta_{t \mid 1}\right)$ each $\beta_{t 11} \in \beta\left(a_{t}, s_{t 11}\right)$. If $t a_{t}$ and the $K_{t+1}$ part of $s_{t+1}$ are not equal, let $\beta\left(a_{t}, s_{t+1}\right)=\left\{\beta_{t+1}\right\}, q^{*}\left(a_{t}, s_{t+1}, \beta_{t+1}\right)=1$, and $y_{t+1}^{\prime}=\mu^{*}\left(a_{t}, s_{t+1}, \beta_{t+1}\right)=$ any element of $\mathscr{Z}$. This last case receives a zero probability weight in the analysis below (see lines (48) and (49)).

As it determines its time $t$ utility-maximizing level of saving, household $j$ will know $y_{i t}$ and $s_{t}, s_{t-1}, s_{t-2}, \ldots$. It will need to forecast signals (from which it can predict factor returns) for the remainder of its life. We suppose it does so with

$$
g\left(S_{t+1}, s_{t}, s_{t, 1}, \ldots\right) \equiv \operatorname{Pr}\left\{s_{t+1} \in S_{1,1} \mid s_{t}, s_{t-1}, \ldots\right\} \quad \text { all } S_{t+1} \in \neq(7)
$$

Let

$$
\sigma_{t} \equiv\left(s_{t}, s_{t-1}, s_{t-2}, \ldots\right)
$$

Then the indirect utility function $v(\cdot)$ for household $j$ comes (recursively) from

$$
v\left(y_{N t}, \sigma_{t}, N ; g\right)=u\left(y_{N t}, N\right)
$$


and, for $1 \leqslant j \leqslant N-1$,

$$
\begin{aligned}
v\left(y_{j t}, \sigma_{t}, j ; g\right)= & \max _{\left\{x \in t: x \leqslant y_{j t}\right.}\left\{u\left(y_{j t}-x, j\right)\right. \\
& +\sum_{s_{t+1} \in t^{\prime}} g\left(\left\{s_{t+1}\right\}, \sigma_{t}\right) \cdot \sum_{\alpha_{t+1} \in \boldsymbol{\alpha}\left(x, s_{t+1}\right)} q\left(x, s_{t+1}, \alpha_{t+1}\right) \\
& \left.\cdot v\left(\mu\left(x, s_{t+1}, \alpha_{t+1}\right),\left(s_{t+1}, \sigma_{t}\right), j+1 ; g\right)\right\}
\end{aligned}
$$

all $t$. Lines (34) and (35) determine the household's set of maximizing values of $x$, say $\psi\left(y_{j t}, \sigma_{t}, j ; g\right)$. If $y_{j t}$ is finitc and non-negative, $\psi(\cdot)$ will be finite and non-empty. As in Section $1, \psi\left(y_{N t}, \sigma_{t}, N ; g\right)=\{0\}$. Define

$$
\Psi\left(y_{t}, \sigma_{t} ; g\right) \equiv \psi\left(y_{1 t}, \sigma_{t}, 1 ; g\right) \times \cdots \times \psi\left(y_{N t}, \sigma_{t}, N ; g\right) .
$$

To see why in general $g\left(S_{t+1}, \ldots\right)$ must depend on the entire history of signals $\sigma_{t}$, suppose $g=g\left(S_{t+1}, s_{t}\right)$. Then $\sigma_{t}, g(\cdot)$, lines (34) and (35), and the fact that every family begins life with no assets imply a set, say $A_{t}^{*}$, of possible values for $a_{t}{ }^{4}$ Let $S_{t+1}^{*} \equiv F\left(A_{t}^{*}, \Lambda\right)$. Careful agents will want $g(\cdot)$ to assign zero probability to signals outside $s_{t+1}$ of $S_{t+1}^{*}$. Since $S_{t+1}^{*}$ depends on $s_{t}, s_{t-1}, \ldots, s_{t-N+2}$ in this case (by construction), to insure consistency between $S_{t+1}^{*}$ and $g\left(S_{t+1}, \cdot\right)$ all $S_{t+1} \in ;(f)$, we will need $g=g\left(S_{t+1}, s_{t}, \ldots, s_{t-N+2}\right)$. If $g(\cdot)$ has this latter form, on the other hand, $S_{t+1}^{*}$ depends on $s_{t}, \ldots, s_{t-2 N+3}$; hence, $g(\cdot)$ should too. Continuing in this way, we can see that only $g=g\left(S_{t+1}, \sigma_{t}\right)$ will avoid objections.

The conclusion itself is an interesting fact.

\section{Result: Households Generally Will Want to Use All Previous Signals in Predicting the Future}

In economic terms, because households lack complete knowledge about the present, they will try to piece together missing information as well as they can from the past. Parenthetically, this process may provide one reason why, say, a linear regression of observed vectors $s_{t}$ on lagged data $s_{t-1}$. $s_{t-2}, \ldots$ often seems to yield non-zero coefficients.

The check derived for $g(\cdot)$ above is not the most exact conceivable: as we derived $A_{t}^{*}$, we disregarded possible restrictions on the interim components of $a_{t-1}$ imposed by $s_{t}$, on $a_{t-2}$ by $s_{t-1}$, etc. To be sure all such information is exploited, we assume that implicity or explicitly each household carries a model $h(\cdot)$ in mind with

$$
h\left(A_{t}, \sigma_{t}\right) \equiv \operatorname{Pr}\left\{a_{t} \in A_{t} \mid \sigma_{t}\right\} \quad \text { all } \quad A_{t}=\mathscr{P}(\mathscr{A})
$$

\footnotetext{
${ }^{4}$ In other words, we can examine every living family's sequence of lifetime maximization problems from birth to the present.
} 
every $t$. For "equilibrium" we demand that $h(\cdot)$ and $g(\cdot)$ make corresponding forecasts with one another and with our behavioral equations.

To establish the continuity and compactness needed for our fixed point results below, we limit the domains of $h(\cdot)$ and $g(\cdot)$ as follows. Suppose our analysis begins at time $t=0$. Let $s_{0}^{*}, s_{-1}^{*}, s_{-2}^{*}, \ldots$ be the actual history of signals. Let $\sigma_{0}^{*} \equiv\left(s_{0}^{*}, s_{-1}^{*}, s_{-2}^{*}, \ldots\right) \equiv\left(s_{0}^{*}, \sigma_{-1}^{*}\right) \equiv\left(s_{0}^{*}, s_{-1}^{*}, \sigma_{-2}^{*}\right) \equiv \ldots$. For $m$, $n \in I$, define

$$
\mathscr{T}^{*}(m, n) \equiv\left\{\left(s_{n}, \ldots, s_{1}, \sigma_{-m}^{*}\right): s_{i} \in \mathscr{F}^{\prime} \text { all } i=1, \ldots, n\right\} .
$$

(Note, for example, that $\mathscr{F}^{*}(0,0)=\left\{\sigma_{0}^{*}\right\}$.) At time 0 , only signal sequences in $\bigcup_{n \geqslant 1} f^{*}(0, n)$ need be considered for the future. In fact, if

$$
F_{\infty}^{*} \equiv \bigcup_{m \geqslant 0, n \geqslant 0} \mathcal{F}^{*}(m, n)
$$

all (past and future) behavior depends solely on sequences in $f^{*}$. Notice that $x_{\infty}^{*}$ is a countable set, a great simplification below-whereas if $x$ is not. ${ }^{5}$

Our sets of forecasting rules $g(\cdot)$ and $h(\cdot)$ are

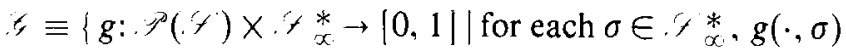

$$
\begin{aligned}
& \text { is a probability measure on } \left.\mathscr{f}\left(f^{\prime}\right)\right\} \text {, }
\end{aligned}
$$

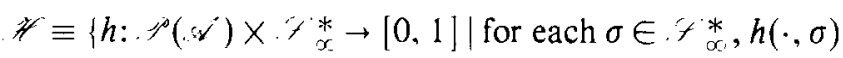

$$
\begin{aligned}
& \text { is a probability measure on } \mathscr{P}(\mathscr{L})\} \text {. }
\end{aligned}
$$

For $g, g^{\prime} \in \xi$, we use the matric

$$
\left\|g-g^{\prime}\right\| \equiv \sup _{m, n \in I}\left(\frac{1}{2}\right)^{n+m} \cdot \max _{s \in, ;, \sigma \in f^{*}(m, n)}\left|g(s, \sigma)-g^{\prime}(s, \sigma)\right| .
$$

For $h, h^{\prime} \in \mathscr{H}$,

$$
\left.\left\|h-h^{\prime}\right\|=\sup _{m, n \in l}\left(\frac{1}{2}\right)^{m+n} \cdot \max _{a \in \mathcal{J}_{, \sigma \in, \mathcal{F}^{*}(m, n)}} \mid h(\{a\}, \sigma)-h^{\prime}(\{a\}, \sigma)\right\} .
$$

If $g, g^{\prime} \in \mathscr{G}$ and $h, h^{\prime} \in \mathscr{H}$, then $\left\|g-g^{\prime}\right\| \leqslant 1$ and $\left\|h-h^{\prime}\right\| \leqslant 1$. These strange-looking functions $\|\cdot\| \rightarrow R$ are metrics for arguments $\sigma \in \mathcal{F}_{\infty}^{*}$ (of $g(\cdot)$ or $h(\cdot))$, but not in general for $\sigma \in \mathscr{F} \times \mathscr{f} \times \cdots$. They make our compactness and continuity results possible. In economic terms, they have the reasonable characteristic of, roughly speaking, weighting the present heavily relative to the distant future and distant past.

We can establish a new version of Lemma 2:

${ }^{5}$ Munkres [15, p. 49] shows ${ }^{*} *$ is countable. In contrast, $\times \times{ }^{\prime} \times \cdots$ has the same cardinality as the set of real numbers in $(0,1)$. 
LEMMA 3. $\quad$ and $\mathscr{H}$ are convex. In the topology induced by $\|\cdot\|$ both are compact.

The appendix supplies a proof.

Our new version of assumption (A7) is

$$
\left(\mathrm{A} 7^{*}\right) \text { If } y_{t} \in \mathscr{Y} g \in \mathscr{G} \text {, and } \sigma_{t} \in \mathscr{F}_{\infty}^{*}, \Psi\left(y_{t}, \sigma_{t} ; g\right) \subset \mathscr{A} \text {. }
$$

The intuition is as in Section 1.

For $g \in \mathscr{\xi}$, the new version of $\Gamma(\cdot)$ is

$$
\begin{aligned}
& \Gamma(g) \equiv\left\{\gamma: \mathscr{O}(\mathscr{N}) \times \mathscr{Y} \times \mathscr{F}_{\infty}^{*} \rightarrow[0,1] \mid\right. \text { for all } \\
&\left(y_{t}, \sigma_{t}\right) \in \mathscr{Y} \times \mathcal{F}_{\infty}^{*}, \gamma\left(\cdot, y_{t}, \sigma_{t}\right) \text { is a probability } \\
& \text { measure on } \mathscr{\mathscr { A }} \text {, and } \\
&\left.\gamma\left(\left\{a_{t}\right\}, y_{t}, \sigma_{t}\right)=0 \text { if } a_{t} \notin \Psi\left(y_{t}, \sigma_{t} ; g\right)\right\} .
\end{aligned}
$$

If $\gamma \in \Gamma(g)$, for every history $\sigma_{t} \in \mathcal{F}_{\infty}^{*}$ (in other words, for every conceivable history) and every current income vector $y_{t} \in \mathscr{Y}, \gamma\left(\cdot, y_{t}, \sigma_{t}\right)$ assigns probability weights to utility-maximizing (see lines (34) and (35)) savings vectors $a_{t}$. Lemma 1 and assumption $\left(\mathrm{A} 7^{*}\right)$ allows us to disregard asset vectors not in $\mathscr{A}$.

We can easily develop a metric for $\Gamma(\mathscr{G})$ by substituting $(a, y) \in \mathscr{Z} \times \mathscr{F}$ for $a \in \mathscr{A}$ in line (43). With the implied topology,

Lemma 4. For $g \in \mathscr{\&}, \Gamma(g)$ is non-empty, convex, and compact. $\Gamma(\cdot)$ is upper semicontinuous on

The Appendix presents a proof.

For $g \in \mathscr{G}$, each $\gamma \in \Gamma(g)$ shows how our economic model takes $y_{t}$, a given history of signals, and a given selection of asset vectors in the case of "ties" into $a_{t}$. We can replace $y_{t}$ with $a_{t-1}$ as follows: for each $g \in \xi$, define

$$
\begin{aligned}
& \Gamma^{*}(g) \equiv\left\{\gamma^{*}: \not \mathscr{P}(\mathscr{y}) \times \mathscr{\mathscr { Y }} \times \mathcal{F}_{\infty}^{*} \rightarrow \mid 0,1\right\} \mid \\
& \text { for some } \gamma \in \Gamma(g) \text { and all }\left(s_{t}, \sigma_{t-1}\right) \in . q_{\infty}^{*}, \\
& a_{t-1} \in \mathscr{W} \text {, and } A_{t} \in \mathscr{\mathscr { O }}(\mathscr{\mathscr { O }}), \gamma^{*}\left(A_{t}, a_{t-1},\left(s_{t}, \sigma_{t-1}\right)\right) \\
& \equiv \sum_{\beta, \in \beta\left(a_{t-1}, s_{t}\right)} q^{*}\left(a_{t-1}, s_{t}, \beta_{t}\right) \\
& \text { - } \left.\gamma\left(A_{t}, \mu^{*}\left(a_{t-1}, s_{t}, \beta_{t}\right),\left(s_{t}, \sigma_{t-1}\right)\right)\right\} \text {. }
\end{aligned}
$$

Notice that for any $\left(a_{t-1}, \sigma_{t}\right) \in \mathscr{A} \times \mathscr{F}_{\infty}^{*}$, each $\gamma^{*} \in \Gamma^{*}(g)$ is a probability measure, $\gamma^{*}\left(\cdot, a_{t-1}, \sigma_{t}\right)$, on $\mathscr{A}$. The preceding lemmas yield 
Lemma 5. For $g \in \mathscr{F}, \Gamma^{*}(g)$ is non-empty, convex, and compact. $\Gamma^{*}(\cdot)$ is upper semicontinuous on

The Appendix provides a proof.

The mapping in terms of which we define our equilibrium, $\Xi$, has two parts. For $S_{t} \in \mathscr{P}(\mathscr{f}), \sigma_{t-1} \in \mathcal{F}_{x}^{*}$, and $h \in \mathscr{F}$, define

$$
d\left(S_{t}, \sigma_{t-1} ; h\right) \equiv \sum_{\left\{\left(a_{t-1}, \lambda_{t}\right\} \in \infty \times \Lambda: F\left\{a_{t-1}, \lambda_{t}\right\} \in S_{t}\right\}} h\left(\left\{a_{t-1}\right\}, \sigma_{t-1}\right) \cdot p\left(\lambda_{t}\right) .
$$

Then for $(g . h) \in G \times, \not H$, define

$$
\Xi^{1}(g, h) \equiv g^{*} \quad \text { with } \quad g^{*}(\cdot) \equiv d(\cdot ; h) .
$$

If $h(\cdot)$ describes households' forecasting rule for next-period asset vectors, rational expectations will require that $\Xi^{1}(g, h)$ and $g$ coincide. This will be our equilibrium consistency condition for $g$ and $h$. Notice that $\Xi^{1}(g, h)$ is single valucd. Lemma 1 , linc (29), and line (41) imply that $\Xi^{1}(\xi \times \not F) \subset$.

Given $h \in \mathbb{F}, s_{t+1} \in \mathcal{H}^{\prime}$, and $\sigma_{t} \in F^{*}$, if $d\left(s_{t+1}, \sigma_{t} ; h\right)=0, s_{t+1}$ is a probability 0 outcome (in terms of agents' predictions). If $d\left(s_{t+1}, \sigma_{t} ; h\right) \neq 0$, we can define agents' forecast of $a_{t}$ conditional on $s_{t+1}$ and $\sigma_{t}$ :

$$
\begin{aligned}
& \bar{h}\left(a_{t}, s_{t+1}, \sigma_{t} ; h\right) \\
& \equiv \sum_{\left(\lambda_{t+1} \in \Lambda: F\left(a_{t} \cdot \lambda_{t+1}\right)=s_{t+1}\right)} h\left(\left\{a_{t}\right\}, \sigma_{t}\right) \cdot p\left(\lambda_{t+1}\right) / d\left(s_{t+1}, \sigma_{t} ; h\right) \\
& \text { all } a_{t} \in \mathscr{x}^{\prime}, s_{t+1} \in \mathcal{F}, \sigma_{t} \in \mathcal{F}^{*} \text {, and } h \in \mathscr{H} \text {. }
\end{aligned}
$$

Coupling this with $\Gamma^{*}(\cdot)$, we have a way in which agents can, with the exacting thoroughness outlined at the beginning of this section, check $h(\cdot)$ : they will want to verify that $h \in \Xi^{2}(g, h)$ where for $(g, h) \in \mathbb{Z} \times$

$$
\Xi^{2}(g, h) \equiv\left\{h^{*}:\left\{0(x) \times 7_{\infty}^{*} \rightarrow[0,1] \mid\right.\right.
$$

$$
\begin{aligned}
& \text { for some } \gamma^{*} \in \Gamma^{*}(g) \text { and all } A_{t+1} \in \mathscr{P}(\mathscr{A}) \\
& \text { and }\left(s_{t+1}, \sigma_{t}\right) \in f^{*}, h^{*}\left(A_{t+1},\left(s_{t+1}, \sigma_{t}\right)\right) \\
& \equiv \sum_{a_{t} \in \mathscr{*}^{*}} \gamma^{*}\left(A_{t+1}, a_{t},\left(s_{t+1}, \sigma_{t}\right)\right) \cdot \bar{h}\left(a_{t}, s_{t+1}, \sigma_{t} ; h\right) \\
& \text { if } d\left(s_{t+1}, \sigma_{t} ; h\right) \neq 0 ; \text { if } d\left(s_{t+1}, \sigma_{t} ; h\right)=0, \\
& \left.h^{*}\left(\cdot,\left(s_{t+1}, \sigma_{t}\right)\right) \text { is an arbitrary probability measure on } \mathscr{\mathscr { t }}\right\}
\end{aligned}
$$

When $d(\cdot) \neq 0, \bar{h}(\cdot)$ defines a conditional probability measure on. Thus, Lemma 1 and assumption $\left(\mathrm{A} 7^{*}\right)$ show $\Xi^{2}(g, h) \subset \mathscr{H}$ all 
$(g, h) \in \mathscr{H}$. Outcomes $\left(s_{t+1}, \sigma_{t}\right) \in \mathscr{F}_{\infty}^{*}$ with $d\left(s_{t+1}, \sigma_{t} ; h\right)=0$ are uninteresting from an economic standpoint, being probability 0 events (in terms of the forecasted evolution of the economy, and in equilibrium the actual evolution as well). Nevertheless, from a mathematical perspective we need to define $\Xi^{2}(\cdot)$ even for $d(\cdot)=0$ and to do so in way leaving $\Xi^{2}(\cdot)$ upper semicontinuous.

\section{Equilibrium}

We are now ready to establish the existence of an equilibrium.

We have a definition corresponding to Section 2.

DEFINITION. $(g(\cdot), h(\cdot))$ constitutes a "stationary equilibrium transition rule" for our new model if $(g, h) \in \mathscr{E} \equiv\{(g, h) \in \mathscr{G} \times \mathscr{H} \mid(g, h) \in$ $\Xi(g, h) \equiv\left(\left(\Xi^{1}(g, h), \Xi^{2}(g, h)\right)\right\}$.

As before, in equilibrium markets always clear (in the sense of (A2) and (A4)) and agents' expectations are rational. Neither $g(\cdot)$ nor $h(\cdot)$ has a time argument; however, we should note that the domain of $g(\cdot)$ is $\mathscr{P}(\mathscr{F}) \times \mathscr{F}_{\infty}^{*}$, the domain of $h(\cdot)$ is $\mathscr{P}(\mathscr{A}) \times \mathscr{P}_{\infty}^{*}$, and $\mathscr{F}_{\infty}^{*}$ depends on $\sigma_{0}^{*}$, an actual history of signals through time 0 . Thus, the nature of the set for $\sigma_{t}$ over which $g(\cdot)$ and $h(\cdot)$ are themselves defined is different for different values of t.

Our basic result is

Proposition 4. Let assumptions (A1)-(A6) and $\left(\mathrm{A} 7^{*}\right)$ hold. Then the set of stationary equilibrium transition rules, $\mathscr{E}$, is non-empty.

The Appendix supplies a proof which is based on a fixed point analysis for $\Xi(\cdot)$ on $\mathscr{G} \times \mathscr{H}$.

From a practical viewpoint we would like to be able to approximate some $(g, h) \in \mathscr{E}$ with functions, say $G(\cdot)$ and $H(\cdot)$, having finite numbers of arguments. Furthermore, we would like to be able to use $(G, H)$ to make forecasts at any date. Although our analysis so far neither establishes nor denies the possibility of such approximations, it does suggest an approach for checking on their existence in particular cases.

The approach is as follows. In order to study approximations which remain valid for all $t$, we construct a metric $*\|\cdot\|^{*}$ independent of our proximity to time 0 : for $g, g^{\prime} \in \mathscr{G}$ and $h, h^{\prime} \in \mathscr{H}$, define

$$
\begin{aligned}
& { }^{*}\left\|g-g^{\prime}\right\|^{*} \equiv \sup _{m, n \in I} \max _{s \in \mathscr{Y}_{, \sigma \in \mathscr{C} *(m, n)}}\left|g(s, \sigma)-g^{\prime}(s, \sigma)\right| \\
& { }^{*}\left\|h-h^{\prime}\right\|^{*} \equiv \sup _{m, n \in I} \max _{a \in \mathscr{A}, \sigma \in \mathscr{F}^{*}(m, n)}\left|h(\{a\}, \sigma)-h^{\prime}(\{a\}, \sigma)\right| .
\end{aligned}
$$


To be able to truncate to a finite list of arguments while introducing only a known maximal error, for any $\theta \in(0,1)$ define subsets

$$
\begin{aligned}
& \mathscr{F} *(\theta) \equiv\left\{g \in \mathscr{G} \mid \text { for all } n \in I \text { and } s_{1}, \ldots, s_{n} \in \mathscr{F}\right. \text {, } \\
& \left.\sup _{\sigma, \sigma^{\prime} \in \gamma^{\prime}}\left|g\left(s_{1}, \ldots, s_{n}, \sigma\right)-g\left(s_{1}, \ldots, s_{n}, \sigma^{\prime}\right)\right| \leqslant \theta^{n}\right), \\
& \mathscr{F} *(\theta) \equiv\left\{h \in \mathscr{F} \mid \text { for all } n \in I \text { and } s_{1}, \ldots, s_{n} \in \mathscr{F} \text { and } a \in \mathscr{F}\right. \text {, } \\
& \left.\sup _{\sigma, \sigma^{\prime} \in ;}\left|h\left(a, s_{1}, \ldots, s_{n}, \sigma\right)-h\left(a, s_{1}, \ldots, s_{n}, \sigma^{\prime}\right)\right| \leqslant \theta^{n}\right\} \text {. }
\end{aligned}
$$

Then we are interested in elements of $\xi^{*}(\theta) \times \mathscr{H}^{*}(\theta)$ close, as measured with $*\|\cdot\|^{*}$, to some $(g, h) \in \mathscr{E}$. Notice that although $\mathscr{H}$ is not compact with $*\|\cdot\|^{*}$,

Lemma 6. For any $\theta \in(0,1), \mathscr{G}^{*}(\theta)$ and $\mathscr{H}^{*}(\theta)$ are compact in the topology induced by *\|. $\| *$.

The Appendix supplies a proof. (Note also that $\mathscr{Y}^{n} \times \mathscr{S}_{\infty}^{*}=\mathscr{F}_{\infty}^{*}$ all $n \in I$ )

Fix any $\theta \in(0,1)$. Suppose $\mathscr{t}^{m} \subset \mathscr{H}^{*}(\theta)$ and $\mathscr{H}^{m} \in \mathscr{H}^{*}(\theta)$ are families of $m$-parameter functions. For all $(g, h) \in \mathscr{G} \times \mathscr{H}$, define

$$
\left.\Delta^{*}(g, h) \equiv \inf _{\left\{g^{\prime}, h^{\prime}\right) \in \Xi(g, h)} \mid *\left\|g-g^{\prime}\right\|^{*}+*\left\|h-h^{\prime}\right\|^{*}\right] .
$$

Suppose for each $m$ we can find $\left(g_{m}, h_{m}\right) \in \mathscr{G}^{m} \times \mathscr{X}^{m}$ with

$$
\lim _{m \rightarrow \infty} \Delta^{*}\left(g_{m}, h_{m}\right)=0 \text {. }
$$

Then there is a subsequence $\left(g_{n}, h_{n}\right)$ of $\left(g_{m}, h_{m}\right)$ and an element $(g, h) \in \mathscr{E}$ such that if "lim" means " $\lim _{n \rightarrow \infty}$ ",

$$
\lim *\left\|g_{n}-g\right\|^{*}=0=\lim *\left\|h_{n}-h\right\|^{*} .
$$

Proposition 5. Let assumptions (A1)-(A6) and (A $\left.7^{*}\right)$ hold. Fix any $\theta \in(0,1)$. Let $\mathscr{G}^{m} \times \mathscr{H}^{m} \subset \mathscr{G}^{*}(\theta) \times \mathscr{H}^{*}(\theta)$ all $m \in I$. Let line (55) hold for some $\left(g_{m}, h_{m}\right) \in \mathscr{G}^{m} \times \mathscr{H}^{m}$ all $m \in I$. Then there exists $(g, h) \in \mathscr{E}$ and $a$ subsequence $\left(g_{n}, h_{n}\right)$ of $\left(g_{m}, h_{m}\right)$ for which line (56) holds.

The appendix provides a proof.

Thus, for some $\theta \in(0,1)$ we try to find $\mathscr{G}^{m} \times \mathscr{H}^{m} \subset \mathscr{G}^{*}(\theta) \times \mathscr{H}^{*}(\theta)$ and $\left(g_{m}, h_{m}\right) \in \mathscr{G}^{m} \times \mathscr{H}^{m}$ such that line (55) holds. Suppose we have been able to do so. Fix any $\varepsilon>0$. Proposition 5 shows there is a subsequence $\left(g_{n}, h_{n}\right)$, an element $(g, h) \in \mathscr{E}$, and an $n^{*}<\infty$ such that $n \geqslant n^{*}$ implies ${ }^{*}\left\|g-g_{n}\right\|^{*}<\varepsilon / 2$ and $*\left\|h-h_{n}\right\|^{*}<\varepsilon / 2$. We can choose $n^{* *}<\infty$ with 
$n^{* *} \geqslant n^{*}$ and $\theta^{n^{* *}}<\varepsilon / 2$. Truncate the list of past-signal arguments for both $g_{n^{* *}}$ and $h_{n^{*}}$ to $n^{* *}$. Call the new functions $G$ and $H$. Then we can use $(G, H)$ to approximate the equilibrium $(g, h) \in \mathscr{E}$ knowing that for any time $t$ and any history $\sigma_{t} \in \mathscr{S}_{\infty}^{*}$, time $t$ one-period forecasts from $(G, H)$ and $(g, h)$ will not differ by more than $\varepsilon$.

Although we could try to relate the ergodic sets of $(G, H)$ to those for $(g, h)$, that is beyond the scope of this paper.

\section{Concluusions}

This paper presents an overlapping generations model subject to random shocks. We assume financial markets are incomplete. Agents may know the entire current distributions of income and wealth, or their information may be limited. In each case we prove the existence of a time-independent probabilistic forecasting rule which, if employed by all agents, leads to an actual stochastic growth path bearing out the predictions. We find that in the case of limited information, current forecasts will generally depend on all previous observable signals.

Although we use relatively few behavioral restrictions, we do require that quantity units and prices come from discrete sets. This complicates our notation somewhat, but in the end we find it greatly facilitates our mathematical arguments. In particular, it enables to establish compactness in various contexts - even (see Sect.4) for a set of functions with infinitely many variables.

In addition to basic existence results, we present several theorems developing approximations. The latter may eventually lead to econometric estimates and/or to policy simulations.

\section{APPENDIX}

\section{Proof of Lemma 1}

Let $l a_{t}>\hat{K}$. Define $f^{*}(K) \equiv \operatorname{Max}_{\lambda \in A} f(K, L, \lambda) . \quad l a_{t+1} \leqslant l y_{t+1} \leqslant f^{*}\left(l a_{t}\right)$. Thus, given (A1), a plot of $f^{*}(\cdot)$ and 45 -degree line, with $k$ on the abscissa, will show there exists $t^{\prime}>t$ such that $l a_{t^{\prime \prime}} \leqslant \hat{K}$ all $t^{\prime \prime}>t^{\prime}$. Let $t a_{t} \leqslant \hat{K}$. Then given (A 1$), t a_{t+1} \leqslant t y_{t+1} \leqslant f^{*}(\hat{K})<\hat{K}$. Continuing in this way, $t a_{t+2} \leqslant \hat{K}$, etc.

\section{Proof of Lemma 2}

Step 1. We show is convex.

Let $g^{\prime}, \quad g^{\prime \prime} \in \mathscr{F} ; \quad \theta \in(0,1) ; \quad y \in \mathscr{Y} ; \quad$ and $\quad A \in \mathscr{P}(\mathscr{A})$. Define $g(\cdot) \equiv \theta \cdot g^{\prime}(\cdot)+(1-\theta) \cdot g^{\prime \prime}(\cdot)$. Then $0 \leqslant \theta \cdot g^{\prime}(A, y)+(1-\theta) \cdot$ 
$g^{\prime \prime}(A, y)=g(A, y) \leqslant \theta+1-\theta=1 ; g(\mathscr{A}, y)=\theta+1-\theta=1$; and, summing over all $a \in A, g(A, y)=\theta \sum g^{\prime}(a, y)+(1-\theta) \sum g^{\prime \prime}(a, y)=\sum\left[\theta \cdot g^{\prime}(a, y)+\right.$ $\left.(1-\theta) \cdot g^{\prime \prime}(a, y)\right]=\sum g(a, y)$. So, $g \in \mathscr{G}$.

Step 2. We show is compact.

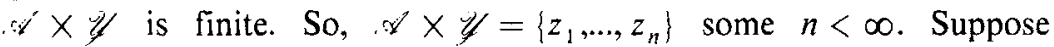
$g_{m} \in$ all $m \in I . g_{m}\left(z_{1}\right) \in[0,1]$ all $m$. So, we can find a subsequence $g_{m(1)}$ of $g_{m}$ converging at $z_{1}$. Similarly, a subsequence $g_{m(2)}$ of $g_{m(1)}$ converges at $z_{2}$. Continue in this way. Then $g_{m(n)}$ converges everywhere on $\mathscr{y}$. Rename it $g_{j}$.

Let "lim" stand for " $\lim _{j \rightarrow \infty}$ ". Define $g\left(z_{i}\right)=\lim g_{j}\left(z_{i}\right)$ all $i=1, \ldots, n$. $n<\infty$ and the construction of $g_{j}$ imply $\lim \left\|g-g_{j}\right\|=0$. Let $y \in \mathscr{y}$ and

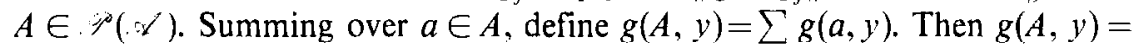
$\sum g(a, y)=\sum \lim g_{j}(a, y)=\lim \sum g_{j}(a, y)=\lim g_{j}(A, y) \in[0,1]$ and $g(\mathscr{x}, y)=\lim g_{j}(\mathscr{x}, y)=\lim 1=1$. So, $g \in \mathcal{C}$. Sequential compactness implies compactness in a metric space (see |15, p. 18|, for example).

\section{Proof of Proposition 1.}

We want to apply the Tychonoff-Kakutani Ky Fan fixed point theorem-see Berge $[2$, p. 251$\}$ - to $I$ defined on $\xi$. We have already established convex and compact, $\Gamma(\xi) \subset \xi$, and $\Gamma(g) \neq \varnothing$ all $g \in \xi$. Let $g \in{ }^{\prime} \%$.

Step 1. We show $\Gamma(g)$ is convex and compact.

Let $\gamma^{\prime}, \quad \gamma^{\prime \prime} \in \Gamma(g) ; \quad \theta \in(0,1) ;$ and $y \in \mathbb{y}$. Define $\gamma(\cdot) \equiv \theta \cdot \gamma^{\prime}(\cdot)+$ $(1-\theta) \cdot \gamma^{\prime \prime}(\cdot)$. Let $a \in, \mathscr{\gamma}$ but $a \notin \Psi(y ; g)$. Then $\gamma(a, y)=\theta \cdot \gamma^{\prime}(a, y)+$ $(1-\theta) \cdot \gamma^{\prime \prime}(a, y)=0+0=0$. Combining this with the analysis in Step 1 of the proof of Lemma 2, we find $\gamma \in \Gamma(g)$. Hence, $\Gamma(g)$ is convex.

Let $\gamma_{m} \in \Gamma(g)$ all $m \in I$ and $\lim _{m \rightarrow \infty}\left\|\gamma_{m}-\gamma\right\|=0$ some $\gamma \in \xi$. Let $y \in y$. Let $a \in, \forall$ but $a \notin \Psi(y ; g)$. Then $\gamma(a, y)=\lim _{m \rightarrow \infty} \gamma_{m}(a, y)=\lim _{m \rightarrow \infty} 0=0$. So, $\gamma \in \Gamma(g)$. Thus, $\Gamma(g)$ is closed. $\Gamma(g) \subset \xi$ and compact. So, $\Gamma(g)$ is compact.

Step 2. We show $\Gamma(\cdot)$ is upper semicontinuous on $\zeta$. To do so, we show that if $U$ is open in $\xi$ with $\Gamma(g) \subset U$, there exists $\varepsilon(g)>0$ such that $g^{\prime} \in$, and $\left\|g-g^{\prime}\right\|<\varepsilon(g)$ imply $\Gamma\left(g^{\prime}\right) \subset U$.

Let $\Gamma(g) \subset U$ with $U$ open. If $y_{t}=y$ and $y_{j}^{*}=y_{j t}=y_{j}$, for each $x$ call the $\{\cdot\}$ expression on the right-hand side of line $(15) \delta(y, x, j ; g)$. Define $\psi^{*}(y, j ; g) \equiv\left\{x \in I \mid x \leqslant y_{j}\right.$ and $\left.x \notin \psi\left(y_{j}, y, j ; g\right)\right\}$. $y$ is finite. Fix any $y=\left(y_{1}, \ldots, y_{N}\right) \in \mathscr{Z}$. Then we can write $\Psi^{*}(y, j ; g)=\left\{z_{1}, \ldots, z_{m}\right\}$ some $m<\infty$-with $m=0$ if $\Psi^{*}(y, j ; g) \neq \varnothing$. Let $x^{*} \in \psi\left(y_{j}, y, j ; g\right)$. Then $\delta\left(y, z_{i}, j ; g\right)<\delta\left(y, x^{*}, j ; g\right)$ all $i=1, \ldots, m$. Thus, line (15) and part (i) of assumption (A5) show there exists $\varepsilon^{*}\left(y, x^{*}, j ; g\right)>0$ such that $g^{\prime} \in \mathbb{Z}$ and $\left\|g-g^{\prime}\right\|<\varepsilon^{*}\left(y, x^{*}, j ; g\right)$ imply $\delta\left(y, z_{i}, f ; g^{\prime}\right)<\delta\left(y, x^{*}, j ; g^{\prime}\right)$ all 
$i=1, \ldots, m$. Let $\varepsilon(g) \equiv \min \left\{\varepsilon^{*}\left(y, x^{*}, j ; g\right) \mid x^{*} \in \psi\left(y_{j}, y, j ; g\right), y \in \mathscr{Y}\right.$, and $1 \leqslant j \leqslant N\}$. Because $\mathscr{Y}$ and $\psi\left(y_{j}, y, j ; g\right)$ are finite, $\varepsilon(g)>0$.

Let $y \in \mathscr{Y}, g^{\prime} \in \mathscr{F}$, and $\left\|g-g^{\prime}\right\|<\varepsilon(g)$. Then $a \notin \Psi(y ; g)$ implies $a \notin \Psi\left(y ; g^{\prime}\right)$. So, $\Psi\left(y ; g^{\prime}\right) \subset \Psi(y ; g)$. Hence, $\Gamma\left(g^{\prime}\right) \subset \Gamma(g) \subset U$.

\section{Proof of Proposition 3.}

Let $\Delta\left(g_{m}\right) \rightarrow 0$ as $m \rightarrow 0$. Since $\xi$ is compact, there exists a subsequence $g_{n}$ of $g_{m}$ and $g \in \mathscr{G}$ such that $\left\|g_{n}-g\right\| \rightarrow 0$ as $n \rightarrow \infty$. Suppose $g \notin \Gamma(g)$. Since we have shown $\Gamma(g)$ is compact, there exists $\varepsilon>0$ with $\Delta(g)=\varepsilon$. Let $U \equiv \bigcup_{g^{*} \in \Gamma(g)} B_{\varepsilon / 3}\left(g^{*}\right){ }^{6}$ Since $\Gamma(\cdot)$ is upper semicontinuous, there exists $\delta>0$ with $\delta<\varepsilon / 3$, and with $g^{\prime} \in \mathscr{G}$ and $\left\|g-g^{\prime}\right\|<\delta$ implying $\Gamma\left(g^{\prime}\right) \subset U$. Then $g^{\prime} \in \mathscr{G}^{\prime}$ and $\left\|g-g^{\prime}\right\|<\delta$ imply $\Delta\left(g^{\prime}\right) \geqslant \varepsilon / 3$. This contradicts $\Delta\left(g_{n}\right) \rightarrow 0$ and $\left\|g_{n}-g\right\| \rightarrow 0$ as $n \rightarrow \infty$. So, $g \in \Gamma(g)$.

\section{Proof of Lemma 3}

We work with

Step 1 (convexity). With $g(\cdot)$ defined over $(S, \sigma) \in \mathscr{P}(\mathscr{S}) \times \mathscr{Y}_{\infty}^{*}$ rather than $(A, y) \in \mathscr{P}(\mathscr{A}) \times \mathscr{Y}$, repeat Step 1 in the proof of Lemma 2.

Step 2 (compactness). The set $\{(m, n): n \in I\}$ is countable. Label its elements $J(k), k \in I$. Let $g_{i} \in \mathscr{F}$ all $i \in I . g_{i}(\cdot) \in[0,1]$, and $\mathscr{f}^{*}(m, n)$ is finite each $(m, n)$. Thus, we can find a subsequence $g_{i(0)}$ of $g_{i}$ converging on $\mathscr{f} \times \mathcal{F}^{*}(J(0)) . g_{i(0)}$ has a subsequence $g_{i(1)}$ converging on $\mathscr{f} \times \mathscr{f}^{*}(J(1))$, and so on. Let $g_{j}$ be the $j$ th element of $g_{i(j)}$. Then $g_{j}$ converges everywhere on $\mathscr{F} \times \mathscr{F}_{\infty}^{*}$ by construction. Let "lim" stand for " $\lim _{j \rightarrow \infty}$ ". Define $g(s, \sigma) \equiv \lim g_{j}(s, \sigma)$ each $\sigma \in \mathscr{F}_{\infty}^{*}$ and $s \in \mathscr{F}$.

Choose any $\varepsilon>0$. Select $n^{*}<\infty$ with $\left(\frac{1}{2}\right)^{n^{\prime}}<\varepsilon$. Let $\mathscr{f}^{* *}=\bigcup_{0 \leqslant m \leqslant n^{*}} \bigcup_{0 \leqslant n \leqslant n^{*}}, \mathscr{S}^{*}(m, n)$. Then $\mathscr{F}^{* *}$ is finite. So, there exists $n^{* *}<\infty$ with $j \geqslant n^{* *}$ implying $\max _{s \in \mathcal{F}, \sigma \in * * *}\left|g(s, \sigma)-g_{j}(s, \sigma)\right|<\varepsilon$. Then $j \geqslant n^{* *}$ implies $\left\|g-g_{j}\right\|<\varepsilon$. Hence the arbitrariness of $\varepsilon$ yields $\lim \left\|g-g_{j}\right\|=0$.

For $(S, \sigma) \in \mathscr{P P}\left(\mathcal{F}^{\prime}\right) \times \mathcal{F}_{\infty}^{*}$, define $g(S, \sigma) \equiv \sum_{s \in S} g(s, \sigma)$. Then summing over $s \in S, g(S, \sigma)=\sum g(s, \sigma)=\sum \lim g_{j}(s, \sigma)=\lim \sum g_{j}(s, \sigma)=$ $\lim g_{j}(S, \sigma) \in[0,1]$ and $g(\mathscr{T}, \sigma)=\lim g_{j}(\mathscr{F}, \sigma)=\lim 1=1$. So, $g \in \mathscr{Z}$. Thus, as in Lemma 2, $\mathscr{G}$ is compact.

\section{Proof of Lemma 4}

Step 1. Let $g \in G . \quad \Gamma(g) \neq \varnothing \quad$ because $\Psi(y, \sigma ; g) \neq \varnothing \quad$ each $(y, \sigma) \in \mathscr{Y} \times \mathscr{F}_{\infty}^{*}$. Let $\gamma^{\prime}, \gamma^{\prime \prime} \in \Gamma(g) ; \theta \in(0,1) ;$ and $\gamma(\cdot) \equiv \theta \cdot \gamma^{\prime}(\cdot)+$ $(1-\theta) \cdot \gamma^{\prime \prime}(\cdot)$. Then $a \notin \Psi(y, \sigma ; g)$ implies $\gamma(a, y, \sigma)=\theta \cdot 0+(1-\theta)$. $0=0$. So, convexity follows as in Step 1 of the proof of Lemma 3. Let

\footnotetext{
${ }^{6} B_{\varepsilon / 3}\left(g^{*}\right)$ stands for the open ball of radius $\varepsilon / 3$ about $g^{*}$.
} 
$\gamma_{i} \in \Gamma(g)$ all $i \in I$ and $\lim _{i \rightarrow \infty}\left\|\gamma_{i}-\gamma\right\|=0$. Then $a \notin \Psi(y, \sigma ; g)$ yields $\gamma(a, y, \sigma)=\lim _{i \rightarrow \infty} \gamma_{i}(a, y, \sigma)=\lim _{i \rightarrow \infty} 0=0$. Compactness then follows as in Step 2 in the proof of Lemma 3.

Step 2. Let $g \in \mathscr{G}, \Gamma(g) \subset U=$ open. We want to show there is $\zeta>0$ such that $g^{\prime} \in G^{\prime}$ and $\left\|g-g^{\prime}\right\|<\zeta$ imply $\Gamma\left(g^{\prime}\right) \subset U$.

For $\gamma \in \Gamma(g)$, define $B_{\varepsilon}(\gamma) \equiv\left\{\right.$ probability measures $\gamma^{*}: \mathscr{P}(\mathscr{A}) \times \mathscr{Y} \times$ $\left.\mathcal{F}_{\infty}^{*} \rightarrow[0,1] \mid \varepsilon>\left\|\gamma-\gamma^{*}\right\|\right\}$. Since $\Gamma(g)$ is compact, there exists $\varepsilon>0$ such that $B_{\varepsilon}(\gamma) \subset U$ all $\gamma \in \Gamma(g)$-see $[9$, p. 158].

Find $n^{*}<\infty$ with $\left(\frac{1}{2}\right)^{n^{*}}<\varepsilon$. Let $\mathscr{f}^{* *} \equiv \bigcup_{0 \leqslant m \leqslant n^{*}, 0 \leqslant n \leqslant n^{*}} \mathscr{F}^{* *}(m, n)$. Then $\mathscr{F}^{* *}$ is finite. So, as in Step 2 in the proof of Proposition 1, there exists $\zeta>0$ such that $g^{\prime} \in \mathscr{F}$ and $\left\|g-g^{\prime}\right\|<\zeta$ imply $\Psi\left(y, \sigma ; g^{\prime}\right) \subset$ $\Psi(y, \sigma ; g)$ all $(y, \sigma) \in \mathscr{Y} \times \mathcal{F}^{* *}$. Thus, $\gamma^{\prime} \in \Gamma\left(g^{\prime}\right), \quad g^{\prime} \in \mathscr{Y}$, and $\left\|g-g^{\prime}\right\|<\zeta \quad$ imply $\quad \gamma^{\prime}(a, y, \sigma)=\gamma^{\prime \prime}(a, y, \sigma) \quad$ some $\quad \gamma^{\prime \prime} \in \Gamma(g) \quad$ all $(a, y, \sigma) \in \mathscr{Y} \times \mathcal{F}^{* *}$. But then $\left\|\gamma^{\prime}-\gamma^{\prime \prime}\right\|<\varepsilon$ by the construction of $f^{* *}$. So, $\gamma^{\prime} \in B_{\varepsilon}\left(\gamma^{\prime \prime}\right) \subset U$. Then the arbitrariness of $\gamma^{\prime} \in \Gamma\left(g^{\prime}\right)$ implies $\Gamma\left(g^{\prime}\right) \subset U$.

So, $g \in \mathscr{G}$ and $\left\|g-g^{\prime}\right\|<\zeta$ imply $\Gamma\left(g^{\prime}\right) \subset U$.

\section{Proof of Lemma 5}

Step 1 (convexity and compactness). Let $g \in \mathscr{H}^{\prime} \cdot \Gamma^{*}(g) \neq \varnothing$ because $\Gamma(g) \neq \varnothing$. Let $\gamma^{*}, \gamma^{* *} \in \Gamma^{*}(g) ; \theta \in(0,1)$; and $\gamma(\cdot) \equiv 0 \cdot \gamma^{*}(\cdot)+(1-\theta)$. $\gamma^{* *}(\cdot)$. Then summing over a finite set of $\beta$ s, $\gamma^{*}(\cdot)=\sum q^{*}(\cdot) \gamma^{\prime}(\cdot)$ and $\gamma^{* *}(\cdot)=\sum q^{*}(\cdot) \cdot \gamma^{\prime \prime}(\cdot)$ some $\gamma^{\prime}, \gamma^{\prime \prime} \in \Gamma(g)$. So, $\gamma(\cdot)=\theta \cdot \sum q^{*}(\cdot) \cdot \gamma^{\prime}(\cdot)+$ $(1-\theta) \cdot \sum q^{*}(\cdot) \cdot \gamma^{\prime \prime}(\cdot)=\sum q^{*}(\cdot) \cdot\left[\theta \cdot \gamma^{\prime}(\cdot)+(1-\theta) \cdot \gamma^{\prime \prime}(\cdot)\right] \in \Gamma^{*}(g)$ because $\Gamma(g)$ is convex.

Let $\gamma_{n}^{*}(\cdot)=\sum q^{*}(\cdot) \cdot \gamma_{n}(\cdot) \in \Gamma^{*}(g)$ all $n \in I$-where $\gamma_{n} \in \Gamma(g)$. Let "Iim" be " $\lim _{n \rightarrow \infty}$ ". Taking subsequences if necessary, the compactness of $\Gamma(g)$ implies there exists $\gamma \in \Gamma(g)$ with $\lim \left\|\gamma_{n}-\gamma\right\|=0$. Define $\gamma^{*}(\cdot) \equiv$ $\sum q^{*}(\cdot) \cdot \gamma(\cdot)$. Then $\gamma^{*} \in \Gamma^{*}(g)$ and $\lim \left\|\gamma^{*}-\gamma_{n}^{*}\right\|=\lim \| \sum q^{*}(\cdot) \cdot \gamma(\cdot)-$ $\sum q^{*}(\cdot) \cdot \gamma_{n}(\cdot) \|=0$ because $\lim \left\|\gamma-\gamma_{n}\right\|=0$. So, $\Gamma^{*}(\cdot)$ is compact.

Step 2 (upper semicontinuity). Let $g \in \mathscr{F}^{*}$ and $\Gamma^{*}(g) \subset U=$ open. As in Lemma 4, there exists $\varepsilon>0$ such that $\gamma^{*} \in \Gamma^{*}(g)$ implies $B_{\varepsilon}\left(\gamma^{*}\right) \subset U$. If $\gamma$, $\gamma^{\prime} \in \Gamma(\mathscr{G})$ and $\left\|\gamma-\gamma^{\prime}\right\|<\varepsilon$, then $\left\|\sum q^{*}(\cdot) \cdot \gamma(\cdot)-\sum q^{*}(\cdot) \cdot \gamma^{\prime}(\cdot)\right\|<\varepsilon$. Lemma 4 shows there exists $\delta>0$ such that $g^{\prime} \in \mathscr{G}$ and $\left\|g-g^{\prime}\right\|<\delta$ imply $\Gamma\left(g^{\prime}\right) \subset \bigcup_{\gamma \in \Gamma(g)} B_{\varepsilon}(\gamma)$.

Let $g^{\prime} \in \mathscr{G}$ and $\left\|g-g^{\prime}\right\|<\delta$. For $\gamma^{\prime} \in \Gamma\left(g^{\prime}\right)$, let $\gamma^{*}(\cdot) \equiv \sum q^{*}(\cdot) \cdot \gamma^{\prime}(\cdot) \in$ $\Gamma^{*}\left(g^{\prime}\right) . \quad \Gamma\left(g^{\prime}\right) \subset \bigcup_{\gamma \in \Gamma(g)} B_{\varepsilon}(\gamma) \quad$ implies there exists $\gamma^{\prime \prime} \in \Gamma(g)$ with $\left\|\gamma^{\prime}-\gamma^{\prime \prime}\right\|<\varepsilon$. Let $\gamma^{* *}(\cdot) \equiv \sum q^{*}(\cdot) \cdot \gamma^{\prime \prime}(\cdot) \in \Gamma^{*}(g)$. Then $\left\|\gamma^{* *}-\gamma^{*}\right\|<\varepsilon$. So, $\gamma^{*} \in B_{\varepsilon}\left(\gamma^{* *}\right) \subset U$. The arbitrariness of $\gamma^{*} \in \Gamma^{*}\left(g^{\prime}\right)$ then shows that $g^{\prime} \in \mathscr{G}$ and $\left\|g-g^{\prime}\right\|<\delta$ imply $\Gamma^{*}\left(g^{\prime}\right) \subset U$. 


\section{Proof of Proposition 4}

As in Proposition 1, we use the Tychonoff-Kakutani-Ky Fan fixed point theorem. We already know $\mathscr{G} \times \mathscr{H}$ convex and compact. $\varnothing \neq \Xi(g, h) \subset \mathscr{G} \times \mathscr{H}$ all $(g, h) \in \mathscr{F} \times \mathscr{H}$, and $\Xi^{1}(\cdot)$ single valued. Clearly $\Xi^{1}(\cdot)$ is continuous. Let $(g, h) \in \mathscr{G} \times \mathscr{H}$.

Step $1\left(\Xi^{2}(g, h) \quad\right.$ convex $)$. Let $h^{*}, \quad h^{* *} \in \Xi^{2}(g, h) ; \quad \theta \in(0,1)$; $(s, \sigma) \in \mathcal{F}^{*} \times \mathcal{f}_{\infty}^{*}=\mathcal{F}_{\infty}^{*}$; and $\hat{h}(\cdot) \equiv \theta \cdot h^{*}(\cdot)+(1-\theta) \cdot h^{* *}(\cdot)$. Suppose $d(s, \sigma ; h)=0 . \quad$ If $\quad A \subset \mathscr{A}, \quad 0 \leqslant \theta \cdot h^{*}(A, s, \sigma)+(1-\theta) \cdot h^{* *}(A, s, \sigma)=$ $\hat{h}(A, s, \sigma) \leqslant 1$; summing over $a \in A, \sum \hat{h}(a, s, \sigma)=\sum\left[\theta \cdot h^{*}(a, s, \sigma)+\right.$ $\left.(1-\theta) \cdot h^{* *}(a, s, \sigma)\right]=\theta \cdot \sum h^{*}(a, s, \sigma)+(1-\theta) \cdot \sum h^{* *}(a, s, \sigma)=\theta$. $h^{*}(A, s, \sigma)+(1-\theta) \cdot h^{* *}(A, s, \sigma)=\hat{h}(A, s, \sigma) ;$ and $\hat{h}(\mathscr{\alpha}, s, \sigma)=$ $\theta+1-\theta=1$. Suppose $d(s, \sigma ; h) \neq 0$. For some $\gamma^{*}, \gamma^{* *} \in \Gamma^{*}(g), h^{*}(\cdot)=$ $\sum \gamma^{*}(\cdot) \cdot \bar{h}(\cdot ; h)$ and $h^{* *}(\cdot)=\sum \gamma^{* *}(\cdot) \cdot \bar{h}(\cdot ; h)$. So, $\hat{h}(\cdot)=\sum\left[\theta \cdot \gamma^{*}(\cdot)+\right.$ $\left.(1-\theta) \cdot \gamma^{* *}(\cdot)\right] \cdot \bar{h}(\cdot ; h)$. Thus, since $\Gamma^{*}(g)$ convex, $\hat{h} \in \Xi^{2}(g, h)$.

Step $2\left(\Xi^{2}(g, h)\right.$ is compact $)$. Let $h_{m}^{*} \in \Xi^{2}(g, h)$ all $m \in I, h^{*} \in \not$, and $\lim _{m \rightarrow \infty}\left\|h_{m}^{*}-h^{*}\right\|=0$. Define $\bar{f}(h) \equiv\left\{(s, \sigma) \in \mathcal{F}^{*} \times \mathcal{F}_{\infty}^{*}=\right.$ $\left.\mathscr{F}_{\infty}^{*} \mid d(s, \sigma ; h)=0\right\}$. Recall $\Xi^{2}(g, h) \subset \not$. Suppose $(s, \sigma) \in \bar{f}(h)$. Then $h^{*} \in \mathscr{H}$ implies $h^{*}(\cdot, s, \sigma)$ is a probability measure on $\mathscr{A}$. Suppose $(s, \sigma) \in, \mathscr{G}_{\infty}^{*}-\overline{\mathscr{f}}(h)$. For each $m$, there exists $\gamma_{m}^{*} \in \Gamma^{*}(g)$ with $h_{m}^{*}(\cdot)=$ $\sum \gamma_{m}^{*}(\cdot) \cdot \bar{h}(\cdot ; h)$. Lemma 5 implies there exist $\gamma^{* *} \in \Gamma^{*}(g)$ and a subsequence $\gamma_{n}^{*}$ of $\underline{\gamma}_{m}^{*}$ such that $\lim _{n \rightarrow \infty}\left\|\gamma_{n}^{*}-\gamma^{* *}\right\|=0$. Define $h^{* *}(\cdot) \equiv \sum \gamma^{* *}(\cdot) \cdot \bar{h}(\cdot ; h)$. Then on $F_{\infty}^{*}-\overline{7}(h), \lim _{n \rightarrow \infty}\left\|h^{* *}-h_{n}^{*}\right\|=0$. Define $\hat{h}(\cdot, s, \sigma)=h^{* *}(\cdot, s, \sigma)$ all $(s, \sigma) \in *^{*}-\bar{h}(h)$ and $\hat{h}(\cdot, s, \sigma)=$ $h^{*}(\cdot, s, \sigma)$ all $(s, \sigma) \in \bar{f}(h)$. Then $\lim _{n \rightarrow \infty}\left\|h_{n}^{*}-\hat{h}\right\|=0$. So, $\hat{h}=h^{*}$. But $\hat{h} \in \Xi^{2}(g, h)$.

Step $3\left(\Xi^{2}(\cdot)\right.$ is upper semicontinuous on $\bar{Z}$. Let $\Xi^{2}(g, h) \subset$ $U=$ open in As in the proof of Lemma 4, there exists $\varepsilon>0$ with $B_{c}\left(h^{*}\right) \subset U$ each $h^{*} \in \Xi^{2}(g, h)$. Let $\overline{\mathscr{f}}(h)$ be as above. In every case, $(s, \sigma) \in f_{\infty}^{*}$.

Lemma 5 shows there exists $\delta^{\prime}>0$ such that $g^{\prime} \in \xi^{\prime}$ and $\left\|g-g^{\prime}\right\|<\delta^{\prime}$ imply for each $\gamma^{*^{\prime}} \in \Gamma^{*}\left(g^{\prime}\right)$ we can choose $\gamma^{*} \in \Gamma^{*}(g)$ with $\left\|\gamma^{*}-\gamma^{* \prime}\right\|<\varepsilon / 2$. Hence, $\left\|\sum \gamma^{* \prime}(\cdot) \cdot \bar{h}(\cdot ; h)-\sum \gamma^{*}(\cdot) \cdot \bar{h}(\cdot ; h)\right\|_{-}<\varepsilon / 2$ all $(s, \sigma) \notin(h)$. Let $h^{* \prime} \in \Xi^{2}\left(g^{\prime}, h\right)$ with $h^{* \prime}(\cdot)=\sum \gamma^{* \prime}(\cdot) \cdot \vec{h}(\cdot ; h)$ all $(s, \sigma) \notin \bar{f}(h)$. Let $h^{*}(\cdot)=\sum \gamma^{*}(\cdot) \cdot \bar{h}(\cdot ; h)$ all $(s, \sigma) \notin \overline{\mathscr{F}}(h)$ and $=h^{* \prime}(\cdot)$ all $(s, \sigma) \in \overline{\mathcal{F}}(h)$. Then $h^{*} \in \Xi^{2}(g, h)$ and $\left\|h^{*}-h^{* \prime}\right\|<\varepsilon / 2$.

For any $\gamma^{*} \in \Gamma(g)$, let $H\left(\cdot ; \gamma^{*}, h\right)$ be any element of $\Xi^{2}(g, h)$ with $H\left(a, s, \sigma ; \gamma^{*}, h\right) \equiv \sum_{a^{\prime} \in \mathscr{W}} \gamma^{*}\left(a, a^{\prime}, s, \sigma\right) \cdot \bar{h}\left(a^{\prime}, s, \sigma ; h\right) \quad$ all $\quad(a, s, \sigma) \in \mathscr{O} \times$ $\bar{F}(h) \#$ where $\overline{\mathscr{F}}(h) \# \equiv \mathscr{Y}_{\infty}^{*}-\overline{\mathscr{F}^{\prime}}(h)$. Let $\left(\frac{1}{2}\right)^{n^{*}}<\varepsilon / 2$. Lines (46) and (48) show there exists $\delta^{\prime \prime}>0$ such that $h^{\prime} \in \mathscr{Z}$ and $\left\|h-h^{\prime}\right\|<\delta^{\prime \prime}$ imply $f^{*}\left(n^{*}, 2 n^{*}\right) \cap \overline{\mathscr{F}}(h) \# \subset \overline{\mathscr{F}}\left(h^{\prime}\right) \#$ and for all $\gamma^{* \prime \prime} \in \Gamma^{*}\left(\xi^{\prime}\right), a \in \mathscr{\mathscr { F }}$, and 
$(s, \sigma) \in \mathscr{F}\left(n^{*}, 2 n^{*}\right) \cap \overline{\mathscr{S}}(h) \#, \quad \mid \sum_{a^{\prime} \in \mathscr{A}}^{\prime} \gamma^{* \prime \prime}\left(a, a^{\prime}, s, \sigma\right) \cdot \bar{h}\left(a^{\prime}, s, \sigma ; h^{\prime}\right)-$ $\sum_{a^{\prime} \in \mathscr{Q}} \gamma^{* \prime \prime}\left(a, a^{\prime}, s, \sigma\right) \cdot \bar{h}\left(a^{\prime}, s, \sigma ; h\right) \mid<\varepsilon / 2$. For all such $h^{\prime}$, given any $H\left(\cdot ; \gamma^{* \prime \prime}, h^{\prime}\right)$ we can find $H\left(\cdot ; \gamma^{* \prime}, h\right)$ equal to $H\left(\cdot ; \gamma^{* \prime \prime}, h^{\prime}\right)$ all $(a, s, \sigma) \in$ $\mathscr{G} \times\left(f^{*}\left(n^{*}, 2 n^{*}\right) \cap\left(\overline{\mathcal{F}}\left(h^{\prime}\right) \#-\overline{\mathcal{F}}(h) \#\right)\right)$. Thus, if $\gamma^{* \prime} \in \Gamma^{*}\left(\xi^{\prime}\right), h^{\prime} \in \mathscr{H}$, and $\left\|h-h^{\prime}\right\|<\delta^{\prime \prime}$, for each $H\left(\cdot ; \gamma^{* \prime}, h^{\prime}\right)$ we can find $H\left(\cdot ; \gamma^{* \prime \prime}, h\right)$ with $\left\|H\left(\cdot ; \gamma^{* \prime \prime}, h^{\prime}\right)-H\left(\cdot ; \gamma^{* \prime \prime}, h\right)\right\|<\varepsilon / 2$.

Let $\delta>0$ and $\delta<\delta^{\prime}, \delta^{\prime \prime}$. Let $\left(g^{\prime}, h^{\prime}\right) \in \mathscr{H} \times \mathscr{H},\left\|g-g^{\prime}\right\|<\delta$, $\left\|h-h^{\prime}\right\|<\delta$, and $\gamma^{* \prime} \in \Gamma^{*}\left(g^{\prime}\right)$. Choose any $H\left(\cdot ; \gamma^{* \prime}, h^{\prime}\right)$. Then there exists $H\left(\cdot ; \gamma^{* \prime}, h\right)$ with $\left\|H\left(\cdot ; \gamma^{* \prime}, h^{\prime}\right)-H\left(\cdot ; \gamma^{* \prime}, h\right)\right\|<\varepsilon / 2$, and there are $\gamma^{*} \in \Gamma^{*}(g)$ and $H\left(\cdot ; \gamma^{*}, h\right)$ with $\left\|H\left(\cdot ; \gamma^{* \prime}, h\right)-H\left(\cdot ; \gamma^{*}, h\right)\right\|<\varepsilon / 2$. So, $\left\|H\left(\cdot ; \gamma^{* \prime}, h^{\prime}\right)-H\left(\cdot ; \gamma^{*}, h\right)\right\|<\varepsilon$. Every element of $\Xi^{2}\left(g^{\prime}, h^{\prime}\right)$ has the form $H\left(\cdot ; \gamma^{* \prime}, h^{\prime}\right)$ some $\gamma^{* \prime} \in \Gamma^{*}\left(g^{\prime}\right)$. Thus, $\Xi^{2}\left(g^{\prime}, h^{\prime}\right) \subset \bigcup_{H \in \Xi^{2}(g, h)}$ $B_{\varepsilon}(H) \subset U$.

\section{Proof of Lemma 6}

We deal with $z^{*}(\theta)$. $*^{*}(\theta)$ is virtually the same. Let "lim" stand for " $\lim _{m \rightarrow \infty} "$.

Suppose $g_{m^{*}} \in G^{*}(\theta)$ all $m^{*} \in I$. Lemma 3 shows there exists $g \in$ and subsequence $g_{m}$ of $g_{m}$. with $\lim \left\|g_{m} \quad g\right\|=0$.

Step 1. We show $g \in \xi^{*}(\theta)$.

Let $s_{1}, \ldots, s_{n} \in f^{\prime}$ and $\sigma, \sigma^{\prime} \in 7^{*}$. Then $\left|g\left(s_{1}, \ldots, s_{n}, \sigma\right)-g\left(s_{1}, \ldots, s_{n}, \sigma^{\prime}\right)\right| \leqslant$ $\left|g\left(s_{1}, \ldots, s_{n}, \sigma\right)-g_{m}\left(s_{1}, \ldots, s_{n}, \sigma\right)\right|+\left|g_{m}\left(s_{1}, \ldots, s_{n}, \sigma\right)-g_{m}\left(s_{1}, \ldots, s_{n}, \sigma^{\prime}\right)\right|+$ $\left|g_{m}\left(s_{1}, \ldots, s_{n}, \sigma^{\prime}\right)-g\left(s_{1}, \ldots, s_{n}, \sigma^{\prime}\right)\right|$. Letting $m \rightarrow \infty$ and noting $g_{m} \in \xi^{*}(\theta)$, $\left|g\left(s_{1}, \ldots ., s_{n}, \sigma\right)-g\left(s_{1} \ldots ., s_{n}, \sigma^{\prime}\right)\right| \leqslant \theta^{n}$. Since $s_{1}, \ldots ., s_{n} \in{ }^{\prime} f$ and $\sigma, \sigma^{\prime} \in \mathcal{F}_{\alpha}^{*}$ are arbitrary, $g \in \xi^{*}(\theta)$.

Step 2. We show $\lim *\left\|g_{m}-g\right\|^{*}=0$.

Fix any $\varepsilon>0$. Choose $n$ with $\theta^{n}<\varepsilon / 4$. Choose $M=M(n, \varepsilon)$ such that $m \geqslant M$ implies $\left|g\left(s_{1}, \ldots, s_{n}, \sigma_{0}^{*}\right)-g_{m}\left(s_{1}, \ldots, s_{n}, \sigma_{0}^{*}\right)\right|<\varepsilon / 2$ all $s_{1}, \ldots, s_{n} \in$. $^{\prime}$. Then $m \geqslant M$ implies that for any $s_{1}, \ldots, s_{n} \in \mathcal{F}^{*}$ and any $\sigma \in \mathcal{F}_{\infty}^{*}$, $\left|g\left(s_{1}, \ldots ., s_{n}, \sigma\right)-g_{m}\left(s_{1}, \ldots, s_{n}, \sigma\right)\right| \leqslant\left|g\left(s_{1}, \ldots, s_{n}, \sigma\right)-g\left(s_{1}, \ldots, s_{n}, \sigma_{0}^{*}\right)\right|+$ $\left|g\left(s_{1}, \ldots, s_{n}, \sigma_{0}^{*}\right)-g_{m}\left(s_{1}, \ldots, s_{n}, \sigma_{0}^{*}\right)\right|+\left|g_{m}\left(s_{1}, \ldots, s_{n}, \sigma_{0}^{*}\right)+g_{m}\left(s_{1}, \ldots, s_{n}, \sigma\right)\right| \leqslant$ $\theta^{n}+(\varepsilon / 2)+\theta^{n}<\varepsilon$. So, lim *\|g $\left\|g_{m}-g\right\|^{*}=0$.

\section{Proof of Proposition 5}

Let line (55) hold. Let "lim" stand for " $\lim _{n \rightarrow \infty}$ ". The proof of Proposition 3 shows there exists $(g, h) \in \mathscr{E}$ and a subsequence $\left(g_{n}, h_{n}\right)$ of $\left(g_{m}, h_{m}\right)$ such that $\lim \left\|g_{n}-g\right\|=0=\lim \left\|h_{n}-h\right\|$. Taking a subsequence of $\left(g_{n}, h_{m}\right)$ if necessary, Lemma 6 shows there exists $\left(g^{*}, h^{*}\right) \in \xi^{*}(\theta) \times \not f^{*}(\theta)$ with $\lim ^{*}\left\|g_{n}-g^{*}\right\|^{*}=0=\lim *\left\|h_{n}-h^{*}\right\|^{*}$. $*\|\cdot\|^{*} \geqslant\|\cdot\|$. So, $\lim \left\|g_{n}-g^{*}\right\|=0=\lim \left\|h_{n}-h^{*}\right\|$. Thus, $g=g^{*}$ on $\mathscr{S}^{*}$ and $h=h^{*} \quad$ on $\quad \mathscr{*} \times x_{\infty}^{*}$. So, $\lim *\left\|g_{n}-g^{*}\right\|^{*}=$ $\lim *\left\|g_{n}-g\right\| *=0=\lim *\left\|h_{n}-h^{*}\right\|^{*}=\lim *\left\|h_{n}-h\right\|^{*}$. 


\section{REFERENCES}

1. K. J. Arrow and F. H. Hahn, "General Competitive Analysis," Holden-Day, San Francisco, 1971.

2. C. Berge, "Topological Spaces" (translated by E. M. Patterson), Oliver \& Boyd, Edinburgh, 1963.

3. T. F. BEWLEY, Existence of equilibria in economies with infinitely many commodities, $J$. Econom. Theory 4 (1972), 514-540.

4. W. Brock and L. Mirman, Optimal economic growth and undertainty: The discounted case, J. Economic Theory 4 (1972), 479-513.

5. J. P. Danthine and J. B. Donaldson, Stochastic properties of fast vs. slow growing economies, Econometrica 49 (1981), 1007-1034.

6. G. Debreu, "Theory of Value," Yale Univ. Press, New Haven, 1973.

7. P. Diamond, National debt in a neoclassical growth model, Amer. Econom. Rev. 55 (1965), 1126-1150.

8. J. Dоoв, "Stochastic Processes," Wiley, New York, 1953.

9. M. C. Gemignanl, "Elementary Topology," Addison-Wesley, Reading, Mass., 1967.

10. J. M. GRANDmont, Temporary general equilibrium theory, in "Handbook of Mathematical Economics, Vol II" (K. J. Arrow and M. D. Intriligator, Eds.). NorthHolland, Amsterdam, 1982.

11. J. Hicks, "Value and Capital," Clarendon Press, Oxford, 1946.

12. F. D. Kydland and E. C. Prescott, Time to build and aggregate fluctuations, Econometrica 50 (1982), 1345-1370.

13. J. LAITNER, The steady states of a stochastic decentralized growth model, J. Econom. Theory 24 (1981), 377-392.

14. R. C. Merton, An asymptotic theory of growth under uncertainty, Rev. Econom. Stud. 42 (1975), 375-394.

15. J. R. Munkres, "Topology," Prentice-Hall, Englewood Cliffs, N.J., 1975.

16. J. F. Muth, Rational expectations and the theory of price movements, Econometrica 29 (1961), 315-335.

17. R. RADNER, Equilibrium under uncertainty, in "Handbook of Mathematical Economics, Vol. II" (K. J. Arrow and M. D. Intriligator, Eds.), North-Holland, Amsterdam, 1982.

18. P. A. Samuelson, An exact consumption-loan model of interest with or without the social contrivance of money, J. Pol. Econom. 66 (1958), 467-482.

19. J. Tobin, Life cycle saving and balanced growth, in "Ten Economic Studies in the Tradition of Irving Fisher" (W. Fellner, Ed.), Wiley, New York, 1967. 